\title{
A survey of water maser emission toward ultracompact HII regions $s^{\star, \star \star}$
}

\author{
P. Hofner ${ }^{1,2}$ and E. Churchwell ${ }^{2}$ \\ 1 I. Physikalisches Institut der Universität zu Köln, Zülpicherstr. 77, D-50937 Köln, Germany \\ 2 Astronomy Department, University of Wisconsin-Madison, 475 North Charter Street, Madison, Wisconsin 53706, \\ U.S.A.
}

Received January 12; accepted April 22, 1996

\begin{abstract}
In this paper we present high resolution images and spectra toward $21 \mathrm{H}_{2} \mathrm{O}$ maser sources in the vicinity of ultracompact (UC) HII regions. This survey provides the basis for future studies with milli-arcsecond resolution, utilizing very long baseline interferometry (VLBI) techniques. Emission from the $616-5_{23}$ masing transition of interstellar $\mathrm{H}_{2} \mathrm{O}$ is observed in the close vicinity of UC HII regions with a median angular distance of 2 .' 9 and a median linear projected distance of $0.1 \mathrm{pc}$ from the continuum peak. We find that for UC HII regions with cometary morphology the water maser emission is located in front of the cometary arc whereas for non-cometary UC HII regions the water masers are often observed projected onto the contours of the ionized gas. Due to the large median distance of the water masers from the I-front of the UC HII region, it is unlikely, that the water masers are formed in the shocked layer of warm molecular gas in the interface between the ionized gas of the UC HII region and surrounding molecular gas which is predicted by the Bow Shock theory of UC HII regions. A comparison with maps in the $\mathrm{NH}_{3}$ inversion transitions shows that in at least 7 cases, the water masers are associated with hot $(T>100 \mathrm{~K})$, dense $\left(n\left(\mathrm{H}_{2}\right) \approx 10^{7} \mathrm{~cm}^{-3}\right)$ molecular clumps. For the UC HII regions G5.89-0.38 and G45.07+0.13 we find spatial and velocity correspondence between water masers and outflowing molecular gas. It is thus likely, that for these sources the $\mathrm{H}_{2} \mathrm{O}$ masers are taking part in the bipolar outflow.
\end{abstract}

Key words: masers - ISM: HII regions - radio lines: ISM

\section{Introduction}

Strong maser action in the $6_{16}-5_{23}$ rotational transition of interstellar $\mathrm{H}_{2} \mathrm{O}$ is observed frequently in star-forming regions and is widely regarded as a signpost of massive star formation. The strong emission and small sizes permit regions as small as $310^{13} \mathrm{~cm}$ to be probed, and the ensemble of $\mathrm{H}_{2} \mathrm{O}$ emission components can be used to investigate the kinematics of the hot, dense molecular gas associated with young stellar objects. While the general connection between star forming activity and $\mathrm{H}_{2} \mathrm{O}$ masers is clearly established, many questions about the details of this association as well as of the physical mechanism which produce $\mathrm{H}_{2} \mathrm{O}$ maser emission, remain.

Send offprint requests to: P. Hofner (Universität zu Köln)

${ }^{\star}$ Based on data collected with the NRAO Very Large Array. The National Radio Astronomy Observatory is operated by Associated Universities, Inc., under contract with the National Science Foundation

${ }^{\star \star}$ Table 3 is also available in electronic form at the CDS via anonymous ftp to cdsarc.u-strasbg.fr (130.79.128.5) or via http://cdsweb.u-strasbg.fr/Abstract.html
Due to the recent advances of theoretical models (e.g. Elitzur et al. 1989; Kaufman \& Neufeld 1995) as well as the availability of NRAO's Very Long Baseline Array (VLBA), it is now possible to improve our understanding of $\mathrm{H}_{2} \mathrm{O}$ masers by increasing the sample of sources studied at milli-arcsecond resolution. As a first step toward this goal, we report here interferometric observations toward $21 \mathrm{H}_{2} \mathrm{O}$ maser sources in the vicinity of ultracompact (UC) HII regions.

UC HII regions represent one of the earliest observable phases in the evolution of massive stars. They are usually associated with dense molecular gas, and the duration of the ultracompact stage has been estimated by Wood \& Churchwell (1989a) to be of order $10^{5} \mathrm{yr}$. The occurrence of distinct morphological types is suggestive of a common mechanism of interaction between the newly formed massive stars and the surrounding molecular gas (Wood \& Churchwell 1989b, hereafter WC). Churchwell et al. 1990 (herafter CWC) observed a large sample of UC HII regions with the MPIfR $100 \mathrm{~m}$ telescope $\left(\mathrm{FWHM}=40^{\prime \prime}\right)$ and detected $\mathrm{H}_{2} \mathrm{O}$ maser emission in $67 \%$ of the sources. This high detection rate shows that $\mathrm{H}_{2} \mathrm{O}$ masers are quite 
common in the vicinity of UC HII regions, thus making them an ideal laboratory to study details of the association between newly formed massive stars and $\mathrm{H}_{2} \mathrm{O}$ maser emission.

In this study we present interferometric observations made with a typical synthesized beam size of 0.4 , which corresponds to a linear size of $0.01 \mathrm{pc}$ or roughly $2000 \mathrm{AU}$ at a distance of $5 \mathrm{kpc}$. This spatial scale is too large to resolve individual maser features, however as our study shows, maser emission in the vicinity of UC HII regions often occurs in several centers of activity which are separated by angular distances that can be resolved by our observations. Thus, besides providing accurate positions for future milli-arcsecond resolution observations, we are able to investigate the relationship between UC HII regions and $\mathrm{H}_{2} \mathrm{O}$ maser emission.

Section 2 of this paper describes the observed sample and details of the observations and data reduction. In this section, we also present images and spectra of the observed $\mathrm{H}_{2} \mathrm{O}$ masers. In Sect. 3, we comment on individual sources and in Sect. 4, the relationship between UC HII regions and $\mathrm{H}_{2} \mathrm{O}$ masers is discussed. We summarize our results in Sect. 5.

\section{Observations and data reduction}

\subsection{The observed sample}

We have selected 21 sources from CWC which show strong emission in the $6_{16}-5_{23}$ masing transition of $\mathrm{H}_{2} \mathrm{O}$. For most of the sources, high resolution radio continuum images were obtained by WC. The ionized gas present in these regions has physical parameters typical of UC HII regions as defined by WC: diameters $\leq 0.1 \mathrm{pc}$, electron densities $\geq 10^{4} \mathrm{~cm}^{-3}$ and emission measures $\geq 10^{7} \mathrm{pc} \mathrm{cm}^{-6}$. To investigate a possible dependence of maser emission on the morphology of the ionized gas, we included several objects of each morphological type as defined by WC. Our sample is thus clearly biased toward resolved, bright UC HII regions with strong maser emission.

\subsection{Data reduction methods}

Observations of the $6_{16}-5_{23}$ transition of interstellar $\mathrm{H}_{2} \mathrm{O}$ were made on December 14, 1991 with the VLA in its B-configuration, which provides baselines between 0.21 and $11.4 \mathrm{~km}$. The intensity scale was set by observation of $3 \mathrm{C} 286$ for which we assumed a total flux density of $2.57 \mathrm{Jy}$ at the frequency of the water line of $22235.08 \mathrm{MHz}$. The synthesized beam FWHM of our observations was about $0^{\prime \prime} 4$. We used a total bandwidth of $3.125 \mathrm{MHz}\left(42 \mathrm{~km} \mathrm{~s}^{-1}\right)$ with 256 channels, which after on-line Hanning smoothing, results in 128 channels with a channel separation of $24.414 \mathrm{kHz}\left(0.329 \mathrm{~km} \mathrm{~s}^{-1}\right)$. The typical integration time per source was 8.5 minutes. Based on the spectra measured by CWC several sources showed water maser fea- tures over an extent larger than our bandwidth. To cover the entire range of emission we observed 8 sources at 2 , and 3 sources at 3 different velocities. The bands were chosen to overlap in order to provide common reference features for self-calibration and also for consistency checks. The instrumental parameters of our observation are summarized in Table 1. In Table 2 we list the sources observed (Col. 1). Columns 2-5 give equatorial coordinates of the radio continuum peak position of the UC HII region, distance and morphological class. Column 6 gives the LSR velocity of the band center and Col. 7 gives the velocity coverage for our observations. Column 8 gives the synthesized beam for each source.

Table 1. VLA instrumental parameters

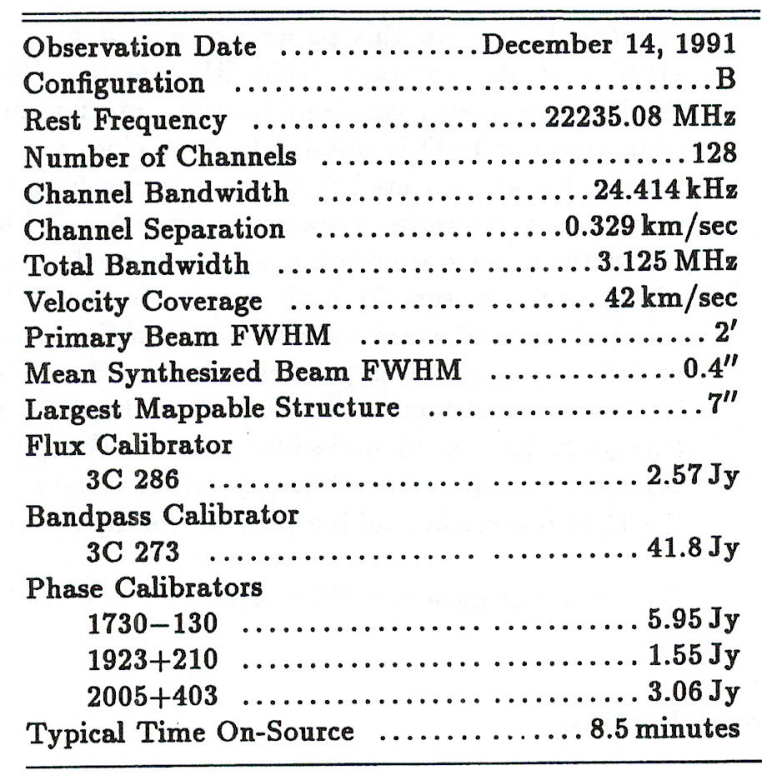

The data were reduced using the NRAO software package AIPS. After inspection and editing, an external calibration was derived from observations of the phase calibrator sources $1730-130,1923+210$ and $2005+403$. A channel with strong maser emission was imaged and used in an iterative self-calibration procedure in order to improve the antenna-based gain and phase solutions. These solutions were subsequently applied to the dataset. In most cases the first (phase-only) iteration was sufficient. The resulting dynamic range in the channel maps is between 300-2000. The calibrated UV-data were imaged and cleaned using the AIPS task MX. The resulting channel maps are $512 \times 512$ pixels with a pixel size of $0{ }^{\prime \prime} 1$. When continuum emission was detected, the line-free channels were averaged to form a continuum map and this map was subtracted from each channel map to create a data-cube containing only the water line.

The entire data-cube was averaged in the UV-plane to create a wide-band data-set. A map covering the entire primary beam of the VLA antennae was then made 
Table 2. Observed sources

\begin{tabular}{|c|c|c|c|c|c|c|c|c|c|}
\hline \multirow[t]{2}{*}{ Source } & \multirow{2}{*}{$\begin{array}{c}\alpha(1950) \\
(\mathrm{h} \min \mathrm{sec})\end{array}$} & \multicolumn{3}{|c|}{$\delta(1950)$} & \multirow{2}{*}{$\begin{array}{c}\mathrm{D} \\
(\mathrm{kpc})\end{array}$} & \multirow[t]{2}{*}{${ }^{\star}$ Class } & \multirow{2}{*}{$\begin{array}{c}\mathrm{V}_{\mathrm{C}} \\
\left(\mathrm{km} \mathrm{s}^{-1}\right)\end{array}$} & \multirow{2}{*}{ 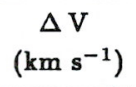 } & \multirow{2}{*}{$\begin{array}{c}\Theta_{\mathrm{SYN}} \\
\left({ }^{\prime \prime}\right)\end{array}$} \\
\hline & & $\left({ }^{\circ}\right.$ & ' & ") & & & & & \\
\hline G5.89-0.38 & $17 \quad 5726.76$ & -24 & 03 & 55.7 & 2.5 & Sh & +44.9 & 111.2 & $0.71 \times 0.33$ \\
\hline G8.67-0.36 & $\begin{array}{lll}18 & 03 & 18.75\end{array}$ & -21 & 37 & 53.2 & 4.6 & $\mathrm{CH}$ & +24.5 & 70.4 & $0.51 \times 0.34$ \\
\hline G9.62+0.19 & $\begin{array}{lll}18 & 03 & 16.20\end{array}$ & -20 & 32 & 03.0 & 5.7 & $\mathrm{Sp}, \mathrm{C}$ & -5.0 & 71.3 & $0.64 \times 0.33$ \\
\hline $\mathrm{G} 10.47+0.03$ & $\begin{array}{lll}18 & 05 & 40.27\end{array}$ & -19 & 52 & 20.8 & 5.8 & MP & +78.6 & 108.5 & $0.48 \times 0.45$ \\
\hline G10.62-0.38 & $\begin{array}{lll}18 & 07 & 27.76\end{array}$ & -19 & 56 & 43.6 & 6.5 & $\mathrm{Sp}$ & -8.0 & 41.4 & $0.51 \times 0.34$ \\
\hline G11.94-0.62 & $\begin{array}{lll}18 & 11 & 04.38\end{array}$ & -18 & 54 & 19.8 & 4.2 & $\mathrm{C}$ & +14.9 & 81.2 & $0.52 \times 0.35$ \\
\hline $\mathrm{G} 12.21-0.10$ & $\begin{array}{lll}18 & 09 & 43.70\end{array}$ & -18 & 25 & 09.0 & 13.5 & $\mathrm{C}$ & +14.9 & 111.2 & $0.49 \times 0.38$ \\
\hline $\mathrm{G} 13.87+0.28$ & $18 \quad 1141.80$ & -16 & 46 & 40.0 & 4.4 & $\mathrm{C}$ & -12.6 & 76.3 & $0.51 \times 0.36$ \\
\hline G19.61-0.23 & $18 \quad 24 \quad 50.27$ & -11 & 58 & 33.4 & 3.5 & $\mathrm{C}, \mathrm{Sp}$ & +39.4 & 76.3 & $0.45 \times 0.36$ \\
\hline G20.08-0.14 & $18 \quad 25 \quad 23.10$ & -11 & 30 & 45.5 & 3.4 & $\mathrm{Sh}, \mathrm{Sp}$ & +45.0 & 41.4 & $0.42 \times 0.33$ \\
\hline G29.96-0.02 & $1843 \quad 27.07$ & -02 & 42 & 36.4 & 7.4 & $\mathrm{C}$ & +93.0 & 41.4 & $0.44 \times 0.41$ \\
\hline $\mathrm{G} 31.41+0.31$ & $\begin{array}{lll}18 & 44 & 59.30\end{array}$ & -01 & 16 & 04.3 & 7.9 & $\mathrm{CH}$ & +88.9 & 77.3 & $0.47 \times 0.41$ \\
\hline $\mathrm{G} 32.80+0.19$ & $\begin{array}{lll}18 & 47 & 57.00\end{array}$ & -00 & 05 & 34.0 & 13.0 & $\mathrm{C}, \mathrm{Sp}$ & +5.0 & 61.5 & $0.59 \times 0.42$ \\
\hline G34.26+0.15 & $\begin{array}{lll}18 & 50 & 46.14\end{array}$ & +01 & 11 & 12.2 & 4.0 & $\mathrm{C}, \mathrm{Sp}_{\mathrm{p}}$ & +52.5 & 96.3 & $0.66 \times 0.41$ \\
\hline G35.20-1.74 & $\begin{array}{lll}18 & 59 & 14.05\end{array}$ & +01 & 09 & 03.0 & 3.1 & C & +40.0 & 41.4 & $0.46 \times 0.40$ \\
\hline G37.55-0.11 & $\begin{array}{lll}18 & 57 & 46.78\end{array}$ & +03 & 58 & 59.8 & 9.9 & $\mathrm{CH}$ & +45.0 & 41.4 & $0.75 \times 0.40$ \\
\hline G43.18-0.52 & $\begin{array}{lll}19 & 09 & 44.79\end{array}$ & +08 & 47 & 03.4 & 4.5 & $\mathrm{CH}$ & +50.0 & 41.4 & $0.47 \times 0.35$ \\
\hline G43.89-0.38 & $\begin{array}{lll}19 & 12 & 02.80\end{array}$ & +09 & 17 & 19.1 & 4.2 & $\mathrm{C}$ & +55.0 & 41.4 & $0.69 \times 0.37$ \\
\hline G45.07-0.13 & $\begin{array}{lll}19 & 11 & 00.40\end{array}$ & +10 & 45 & 42.9 & 6.0 & $\mathrm{Sp}$ & +60.0 & 41.4 & $0.61 \times 0.37$ \\
\hline G50.32+0.68 & $19 \quad 19 \quad 11.40$ & +15 & 38 & 37.2 & 8.7 & $\mathrm{Sp}$ & +25.0 & 41.4 & $0.65 \times 0.35$ \\
\hline \multirow[t]{2}{*}{ G75.78-0.34 } & $20 \quad 19 \quad 52.00$ & +37 & 17 & 01.8 & 4.1 & $\mathrm{C}$ & -10.0 & 71.3 & $0.52 \times 0.30$ \\
\hline & ^Class: & $\begin{array}{l}\mathrm{Sh} \\
\mathrm{C}- \\
\mathrm{CH} \\
\mathrm{Sp} \\
\mathrm{MP}\end{array}$ & & herical & Peake & olved & & & \\
\hline
\end{tabular}

to search for features with large offsets from the phase center of our observations. Due to the spectral dilution, the resulting limit of detection in this map is $\approx 1 \mathrm{Jy}$ for an individual maser feature. The flux densities of features at distances exceeding $20^{\prime \prime}$ from the phase-tracking center were corrected using a gaussian response function for the primary beam of the VLA antenna.

The channel maps were inspected for maser features and the position and intensity of the features were recorded. For each distinguishable maser in the field of view, a spectrum was taken at the position of the pixel with maximum emission. To derive the exact position and intensity, a gaussian was fitted to the channel with maximum emission of each spectral feature. In cases where different maser spots are blended, the intensity was derived from a fit of multiple gaussian components. No fit in the spectral domain was undertaken.

A typical noise level in our channel maps is $30 \mathrm{mJy} /$ beam; we recorded features down to a limiting intensity of $\approx 0.2 \mathrm{Jy} /$ beam. In a few cases when very strong features were present, the noise in the channel maps was considerably higher, so that weak sources could have been missed in these cases.

Assuming a size much smaller than our typical synthesized beam, FWHM of 0.4 , we estimate for the weakest features a relative positional accuracy of about 0 "' 1 . Since the positional accuracy obtained by gaussian fitting scales inversely with the signal-to-noise ratio of the feature, the theoretical positional accuracy of most features is much higher; in most cases structure in the beam is more likely to be the limiting factor. Also, due to the self-calibration process all positions are tied to the position of the reference feature.

In Table 3 we list the observed water maser features. For each source we have grouped together as clusters, spectral features which cannot be separated in the map, i.e. which are at distances smaller than the synthesized beam. For each cluster we list the position, velocity and intensity of the strongest feature (Cols. 3-6) and Col. 7 gives the velocity range over which emission is observed in a given cluster. 


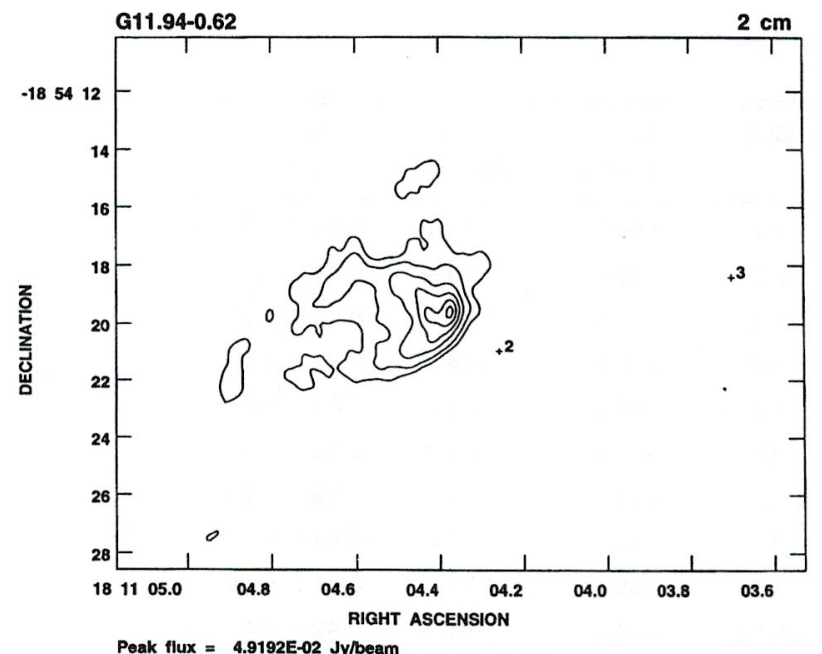

Peak flux $=4.9192 E-02$
Levs $=4.9192 E-04$$(-10,10,20,40,60,80,95)$

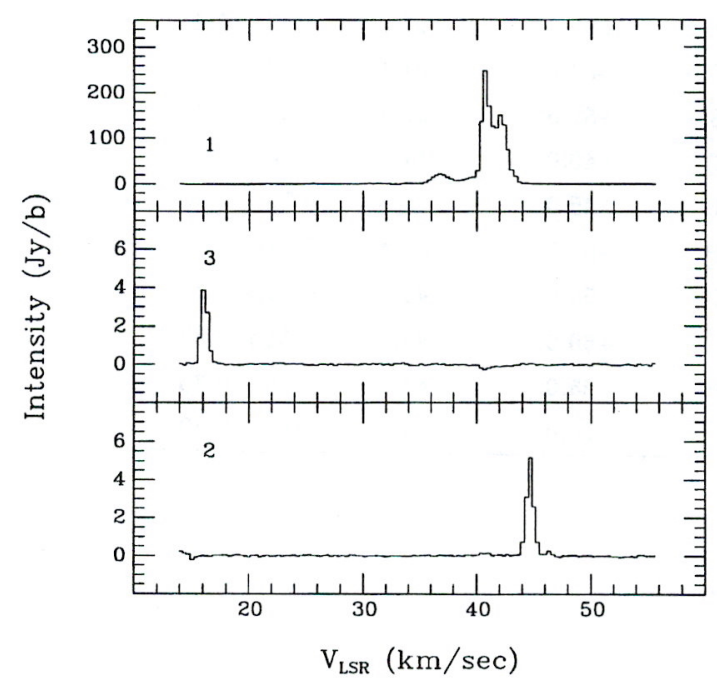

Fig. 1. a) $2 \mathrm{~cm}$ continuum map of the UC HII region G11.94-0.62 from WC. The crosses show the position of $\mathrm{H}_{2} \mathrm{O}$ maser features of clusters 2 and 3 . Cluster 1 is outside the area shown. b) $\mathrm{H}_{2} \mathrm{O}$ maser spectra of clusters 1,2 and 3 , taken at the pixel closest to the cluster center

In Figs. 1-21, we show the location of each spectral feature as crosses relative to the ionized gas of the UC HII regions in the following order: Figs. 1-7 show cometary UC HII regions, Figs. 8 and 9 have multiple components, at least one of which has a cometary morphology, and Figs. 10-19 show UC HII regions with non-cometary morphology. Unless otherwise noted, the continuum maps are from WC. Note that some features were detected at large angular distances from the UC HII region, so that their position cannot be shown on the continuum maps. Below the continuum maps, we show spectra which were read out at the pixel closest to the cluster center. In some cases when a very strong maser feature is present, residual phase errors result in artifacts in the spectra of nearby features.

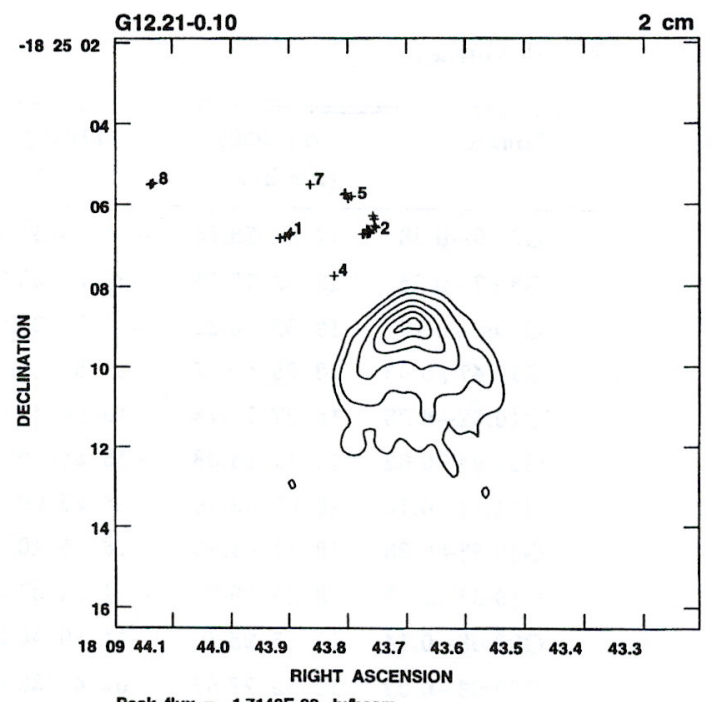

Peak flux $=1.7148 \mathrm{E}-02$ Jy/beam
Lovs $=1.7148 \mathrm{E}-04 \cdot(-10,10,20,40,60,80,95)$

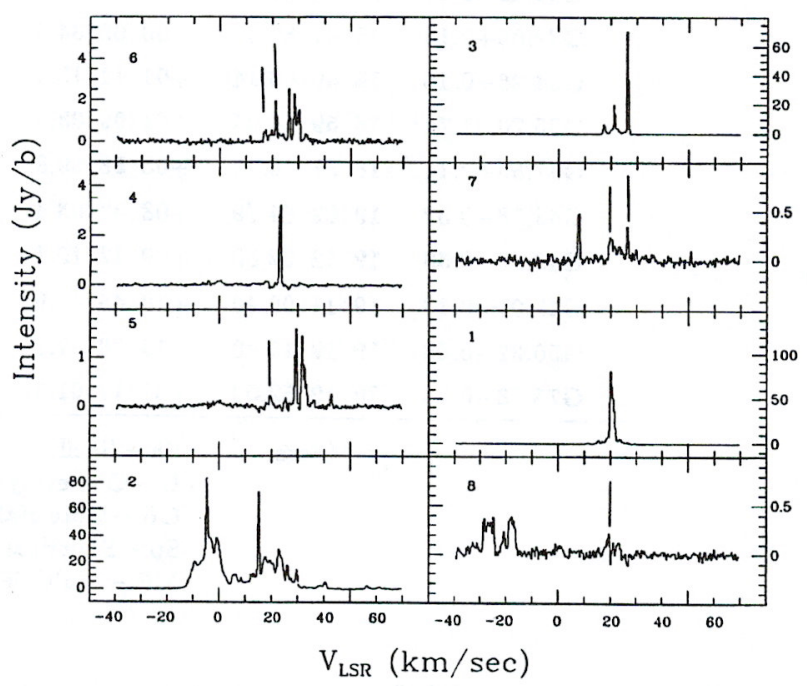

Fig. 2. Results for G12.21-0.10. Captions are as in Fig. 1. Vertical lines in Fig. 2b mark spectral artifacts

For example, the strong features of maser cluster No. 1 in G19.61-0.23 (see Fig. 3) cause the negative artifacts seen in the spectra of clusters Nos. 3, 4 and 5 in this source. We have marked artifacts in the spectra by vertical lines. A complete list of positions and fluxes for each spectral feature can be obtained from the authors.

\section{Comments on individual sources}

Several of the maser sources observed in this survey show interesting features such as symmetric line profiles, elongated structures and velocity gradients. Detailed modeling of these sources is beyond the scope of this survey and will be attempted for selected sources in a subsequent paper. Below we comment on some of the more interesting sources. 

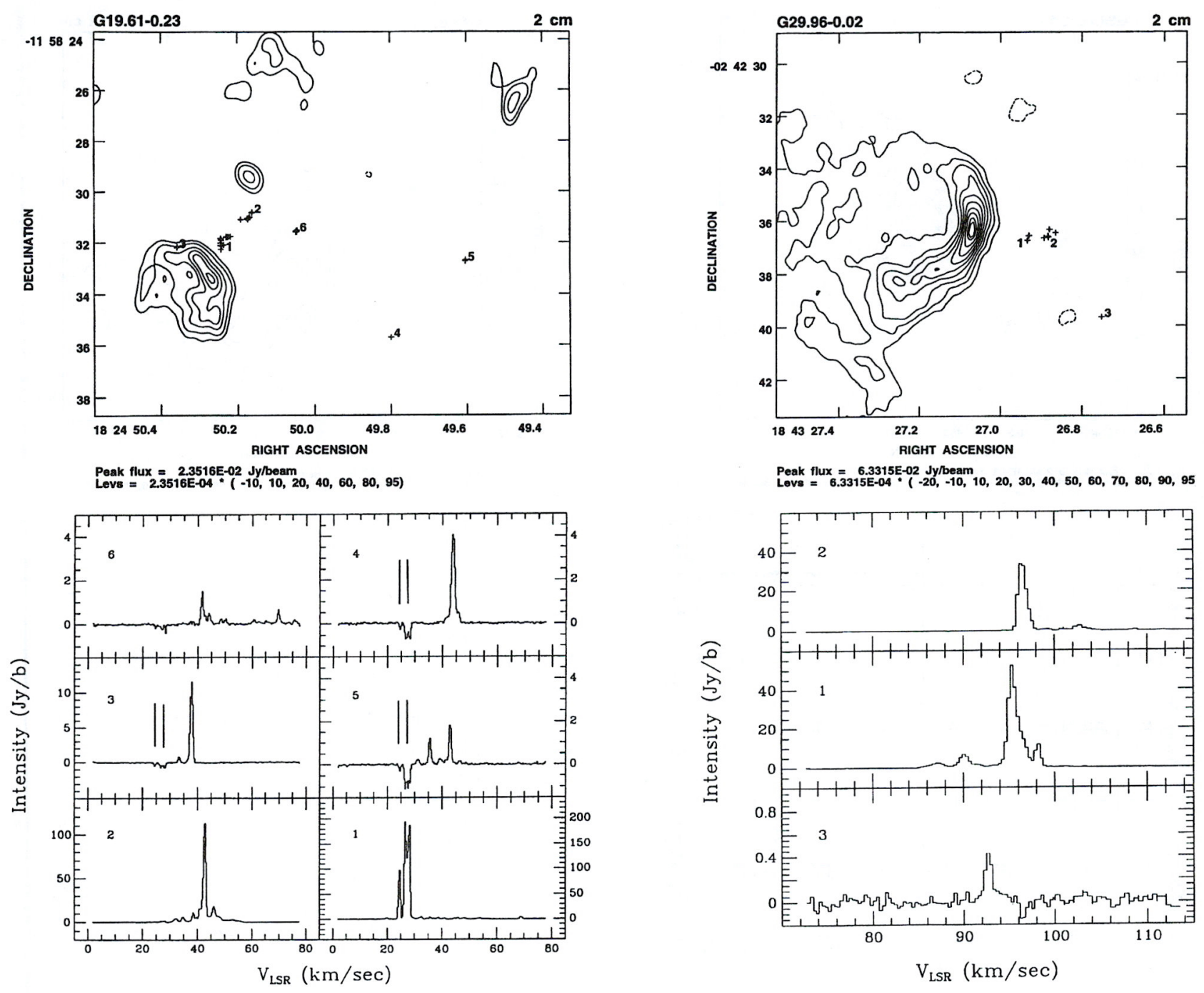

Fig. 3. Results for G19.61-0.23. Captions are as in Fig. 1

Fig. 4. Results for G29.96-0.02. Captions are as in Fig. 1

\section{1. $G 5.89-0.38$}

The shell type UC HII region G5.89-0.38 is the source of one of the most luminous bipolar molecular outflows in the galaxy. The CO observations of Harvey \& Forveille (1988) showed that the flow is oriented in the East-West direction. However, in a subsequent paper by Cesaroni et al. (1991), the orientation was reported to be North-South, based on a map of the $J=3-2$ transition of $\mathrm{C}^{34} \mathrm{~S}$. The positions of the $\mathrm{H}_{2} \mathrm{O}$ masers toward G5.89-0.38 (Fig. 10) are distributed in a North-South orientation and if one adopts the brightest maser line in each cluster as representative of the radial velocity, a clear velocity trend can be seen ranging from close to $80 \mathrm{~km} \mathrm{~s}^{-1}$ for the northernmost component to about $-4 \mathrm{~km} \mathrm{~s}^{-1}$ for the southernmost component. This is particularly clear for clusters Nos. 1, 2 and 3. Several emission components in the $\mathrm{H}_{2} \mathrm{O}$ maser line at velocities outside of our velocity coverage have also been

reported: an emission component at $V_{\mathrm{LSR}}=-61 \mathrm{~km} \mathrm{~s}^{-1}$ had been observed by Genzel \& Downes (1977), but was not detected by Zijlstra et al. (1990); recently, emission at about $-20 \mathrm{~km} \mathrm{~s}^{-1}$ was observed by Acord et al. (1994, private communication). The sense and orientation of the water masers mapped is the same as for the bipolar flow mapped in $\mathrm{C}^{34} \mathrm{~S}$ by Cesaroni et al. (1991). Also, if one takes into account the additional velocity components mentioned above we note that the velocity range seen in the $\mathrm{H}_{2} \mathrm{O}$ masers is $\approx 140 \mathrm{~km} \mathrm{~s}^{-1}$ which is almost identical to the velocity extent of the flow as seen in $\mathrm{CO}$ by Harvey \& Forveille (1988). Hence, there is strong evidence that in G5.89-0.38 the $\mathrm{H}_{2} \mathrm{O}$ masers are taking part in the bipolar molecular outflow traced by $\mathrm{C}^{34} \mathrm{~S}(3-2)$. 


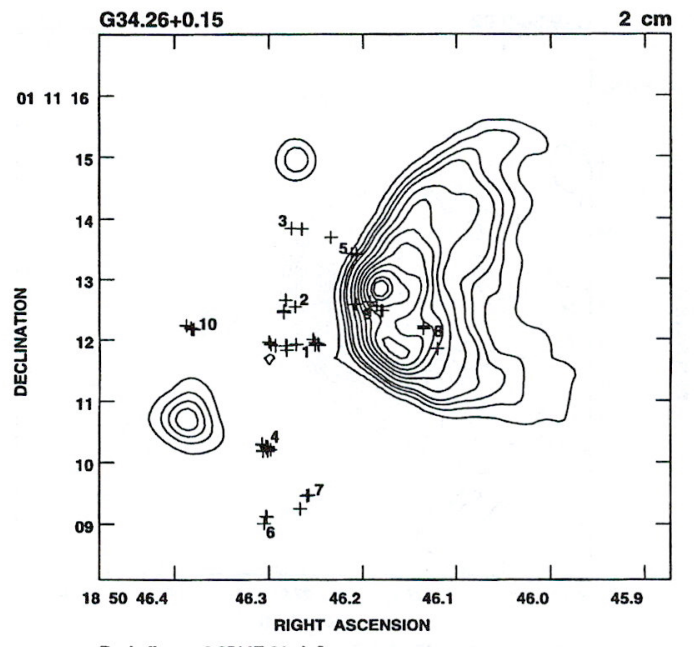

Peak flux $=2.9514 \mathrm{E}-01$ Jy/beam
Levs $=2.9514 E-03 \cdot(-10,5,10,15,20,30,40,50,60,70,80,90,95)$

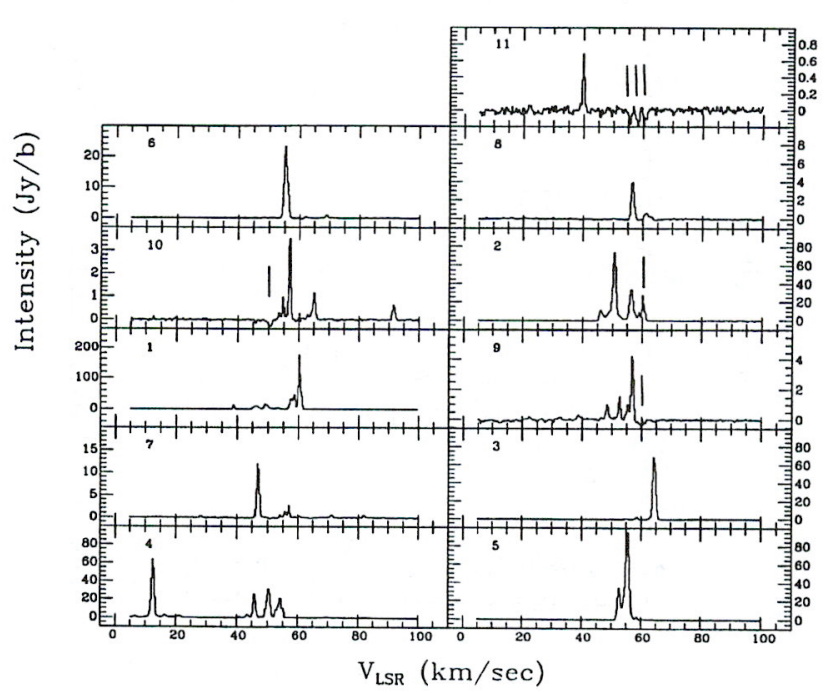

Fig. 5. Results for G34.26+0.15. Captions are as in Fig. 1

\section{2. $G 9.62+0.19$}

The $\mathrm{H}_{2} \mathrm{O}$ maser emission in G9.62+0.19 (see Fig. 8) is located along a narrow, linear structure of length $0.6 \mathrm{pc}$. Along this line, several other maser species as well as hot $\mathrm{NH}_{3}$ and very compact continuum sources are also observed (see Hofner et al. 1994 and references therein). This line of star forming activity occurs along a ridge and is embedded in a massive molecular cloud core of mass $\approx 1000$ $M_{\odot}$, in which at least three centers of recent star formation are present (Hofner et al. 1996).

\section{3. $G 10.47+0.03$}

Three strong maser clusters are detected in the immediate surroundings of the UC HII region G10.47+0.03B (see Fig. 12). The $\mathrm{NH}_{3}(4,4)$ observations of Cesaroni et al. (1994, hereafter CCHWK) show that the UC HII region is located at the center of a spherical molecular clump with

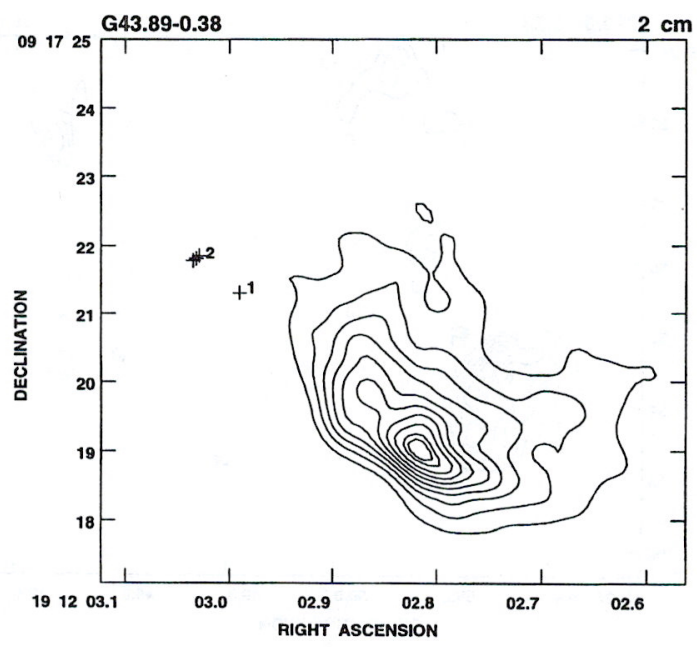

Peak flux $=2.2312 \mathrm{E}-02$ Jy/beam
Levs $=2.2312 \mathrm{E}-04 \times(-10,10,20,30,40,50,60,70,80,90,95)$

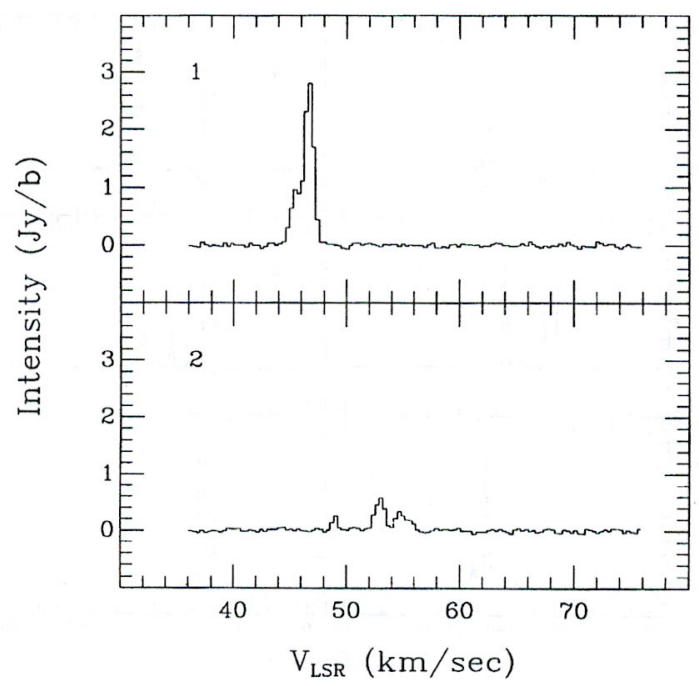

Fig. 6. Results for G43.89-0.38. Captions are as in Fig. 1

an average gas temperature of $200 \mathrm{~K}$ and an average hydrogen density of $n_{\mathrm{H}_{2}} \approx 10^{7} \mathrm{~cm}^{-3}$. Blue shifted ammonia absorption indicates that a substantial amount of molecular gas is expanding. The ionized gas at the center of the molecular clump is still optically thick at $1.3 \mathrm{~cm}$ indicating a very high electron density. The water maser clusters (Fig. 12) appear projected onto the molecular gas traced by $\mathrm{NH}_{3}$ and may be part of an expanding molecular envelope, which is heated by the very young massive star responsible for the ionization in the center of the clump.

\section{4. $G 10.62-0.38$}

In a series of papers Keto, Ho \& Haschick and coworkers investigated the dense molecular gas associated with the Core-Halo UC HII region, using the $\mathrm{NH}_{3}$ inversion lines as a probe of the physical conditions and kinematics in the region. The observed velocity field is interpreted by these 


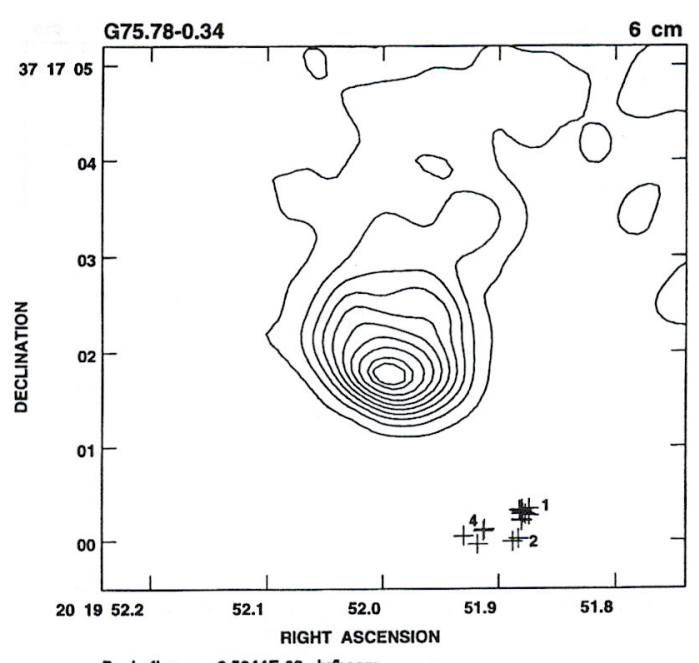

Peak flux $=6.5844 \mathrm{E}-03$ Jy/beam
Levs $=6.5844 \mathrm{E}-05 \mathrm{*}(-20,-10,10,20,30,40,50,60,70,80,90,95)$

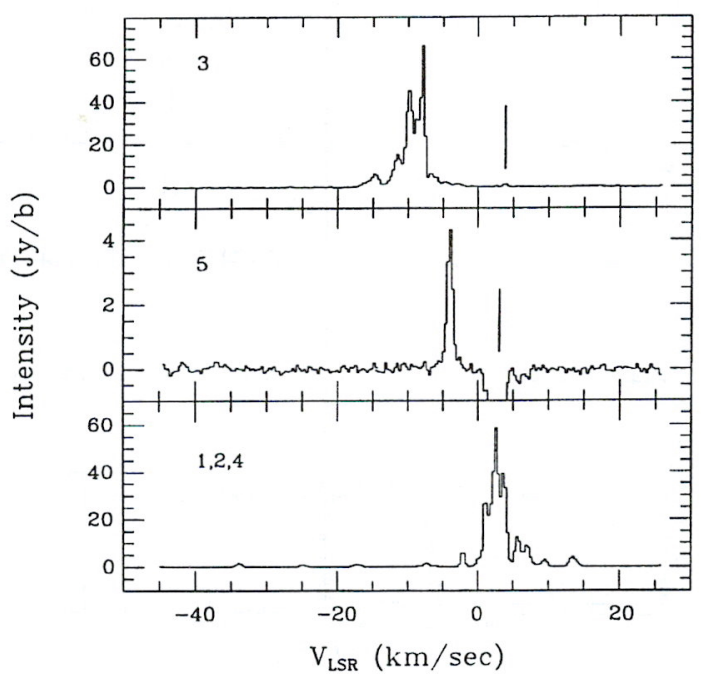

Fig. 7. Results for G75.78-0.34. Captions are as in Fig. 1

authors as a remnant infalling molecular envelope onto a massive young star. The molecular gas is also seen to be rotating and undergoing spin-up as it approaches the UC HII region (e.g. Keto et al. 1987, 1988). We detect strong maser emission coincident with the $\mathrm{NH}_{3}$ gas to the northwest of the UC HII region which forms a linear structure of projected length $\approx 0.04 \mathrm{pc}$, oriented perpendicular to the rotation axis of the molecular gas (clusters 1-4, Fig. 13).

\section{5. $G 13.87+0.28$}

G13.87+0.28 is a cometary HII region located at a distance of $4.4 \mathrm{kpc}$. Garay et al. 1993 obtained 2, 6 and $20 \mathrm{~cm}$ continuum observations toward this source with the VLA. Their work showed that G13.87+0.28 has a diameter of $0.43 \mathrm{pc}$, electron density $2.510^{3} \mathrm{~cm}^{-3}$ and emission measure $4.110^{6} \mathrm{pc} \mathrm{cm}^{-6}$ and thus does not qualify as an ultracompact $\mathrm{HII}$ region. The $\mathrm{H}_{2} \mathrm{O}$ maser spectrum toward
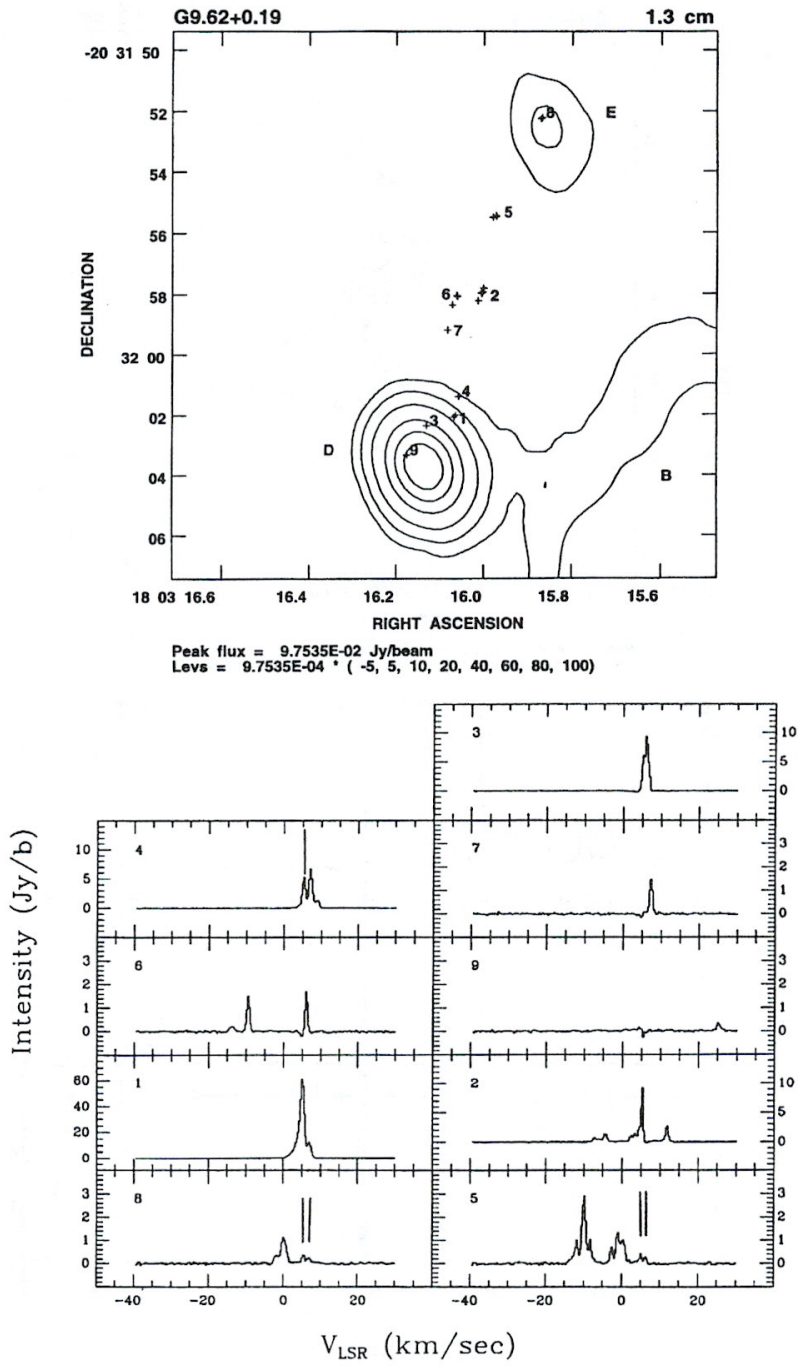

Fig. 8. Results for G9.62+0.19. Captions are as in Fig. 1. The continuum image is from CCHWK. Continuum components B, D and E (using the nomenclature of Garay et al. 1993) are indicated. Component $\mathrm{B}$ extends to the west and has a cometary morphology (see CCHWK)

G13.87+0.28 is shown in Fig. 19. Emission is seen over a velocity extent larger than our bandwidth so that the velocity extent quoted in Table 3 is a lower limit. With the given spatial resolution all velocity features originate from a single cluster, located close to the $6 \mathrm{~cm}$ radio continuum peak.

\subsection{G29.96-0.02}

The $\mathrm{H}_{2} \mathrm{O}$ maser emission toward the cometary UC HII region in G29.96-0.02 is located just ahead of the apex of the ionized gas. Recent high resolution observations of the $\mathrm{NH}_{3}(4,4)$ line by CCHWK show that the maser emission is coincident with a molecular clump with $T \approx 100 \mathrm{~K}$ and $n_{\mathrm{H}_{2}} \approx 10^{7} \mathrm{~cm}^{-3}$. The $\mathrm{H}_{2} \mathrm{O}$ maser spectra at the two 

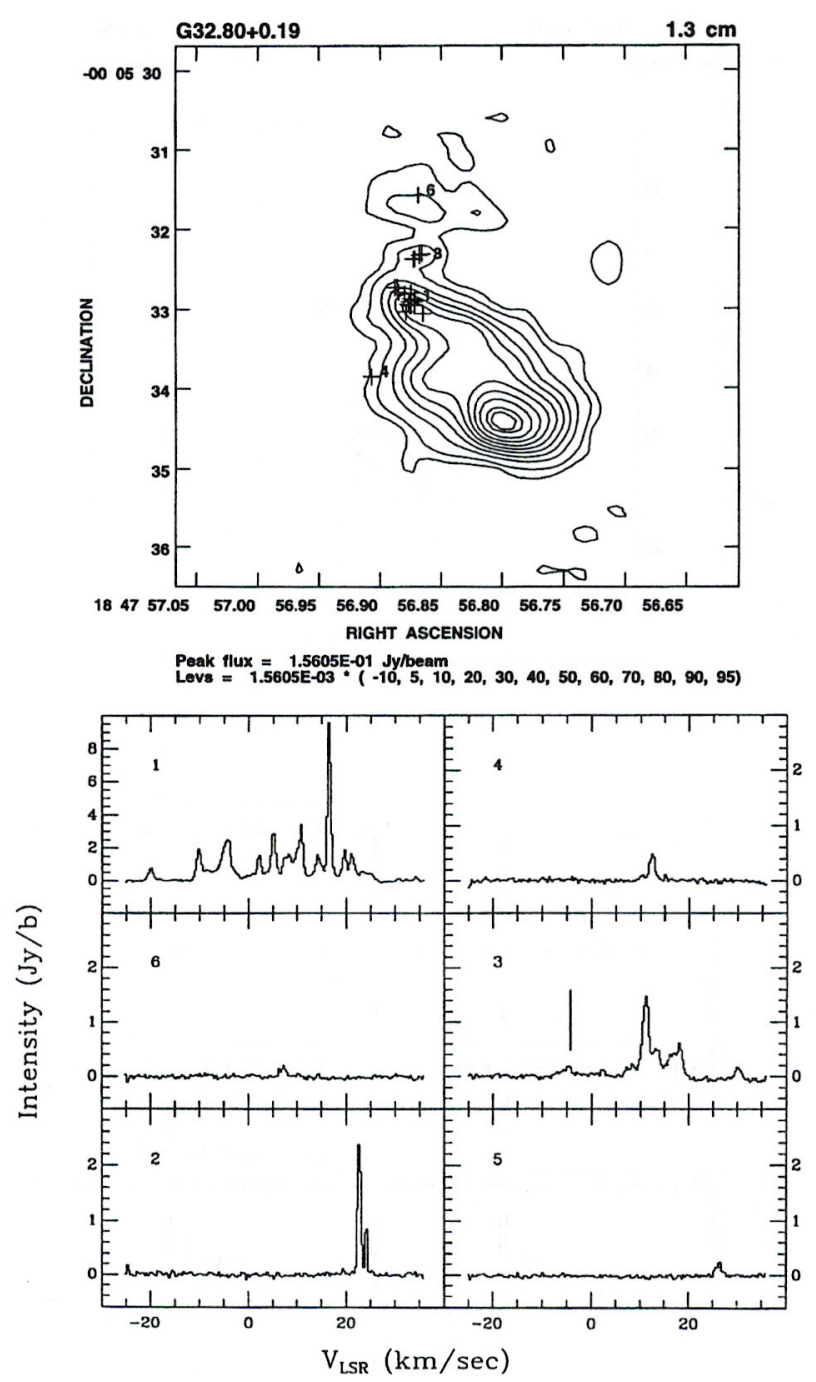

Fig. 9. Results for G32.80+0.19. Captions are as in Fig. 1. The continuum image was derived from line free channels in the $\mathrm{H}_{2} \mathrm{O}$ maser observations. Due to the narrow bandwidth of the maser observations only the brightest sources are seen in our map. See KCW for a continuum map of higher signal-to-noise ratio

clusters in front of the ionized arc show an interesting symmetry (Fig. 4): For both maser spots the strongest emission occurs at velocities close to the velocity of the molecular clump. The eastern maser clump shows additional blue shifted features whereas only red shifted features are found at the position of the western maser cluster, so that the profiles at the two clusters are almost mirror images of each other. A similar spectral behavior is found for the water masers associated with NGC 2071 IRS1 by Tofani et al. 1995 and is interpreted by these authors as a bipolar molecular flow. Alternatively, in the model suggested by Churchwell (1993) the profiles are explained by maser emission from a rotating molecular disk or torus surrounding a young stellar object.

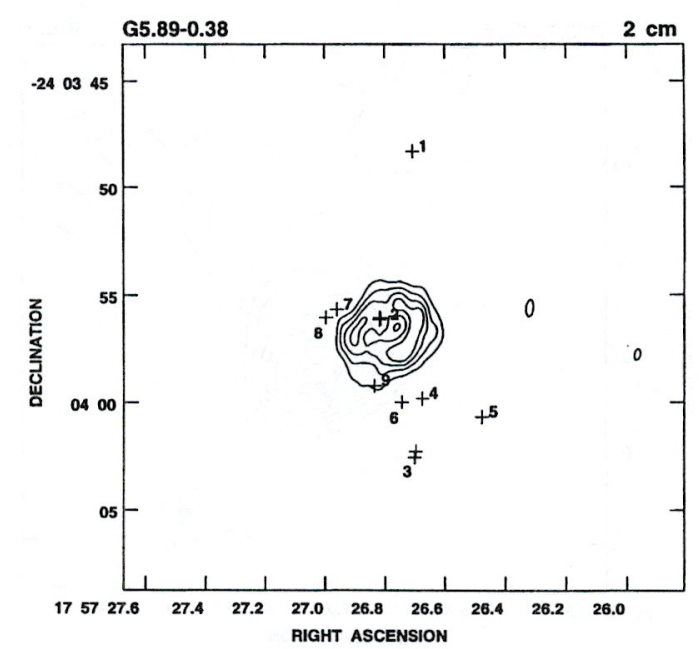

Peak flux $=2.8653 E-01$ Jy/boam
Levs $=2.8653 E-03 \div-(-20,20,40,60,80,100)$

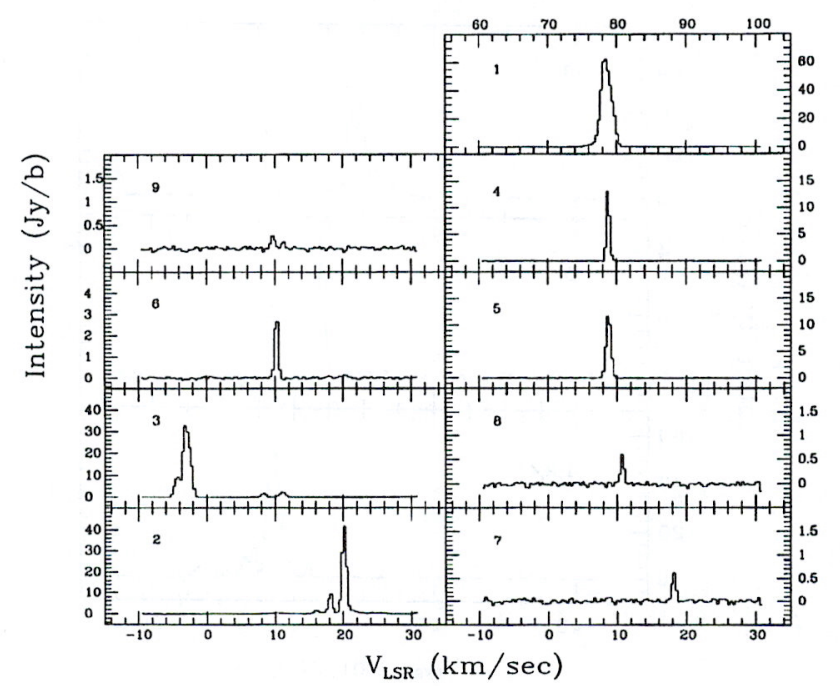

Fig. 10. Results for G5.89-0.38. Captions are as in Fig. 1. Note the different velocity scale for the spectrum of maser feature No. 1 in Fig. 10b

\section{7. $G 31.41+0.31$}

The water masers in G31.41+0.31 are coincident with a hot molecular clump with temperature and density similar to the molecular clump in G10.47+0.03 (CCHWK). Recent interferometric observations toward G31.41+0.31 have revealed a bright $3 \mathrm{~mm}$ continuum source coincident with the molecular clump as well as an unusually large velocity gradient observed in the $\mathrm{CH}_{3} \mathrm{CN}(6-5)$ line (Cesaroni et al. 1994). This velocity gradient is also visible in the $\mathrm{OH}$ masers observed at the position of the molecular clump (Gaume \& Mutel 1987) but is not clearly identified in the water maser spectra in Fig. 15. The data are consistent with $\mathrm{H}_{2} \mathrm{O}$ masers embedded in the molecular clump in which a massive stellar object has recently formed. 

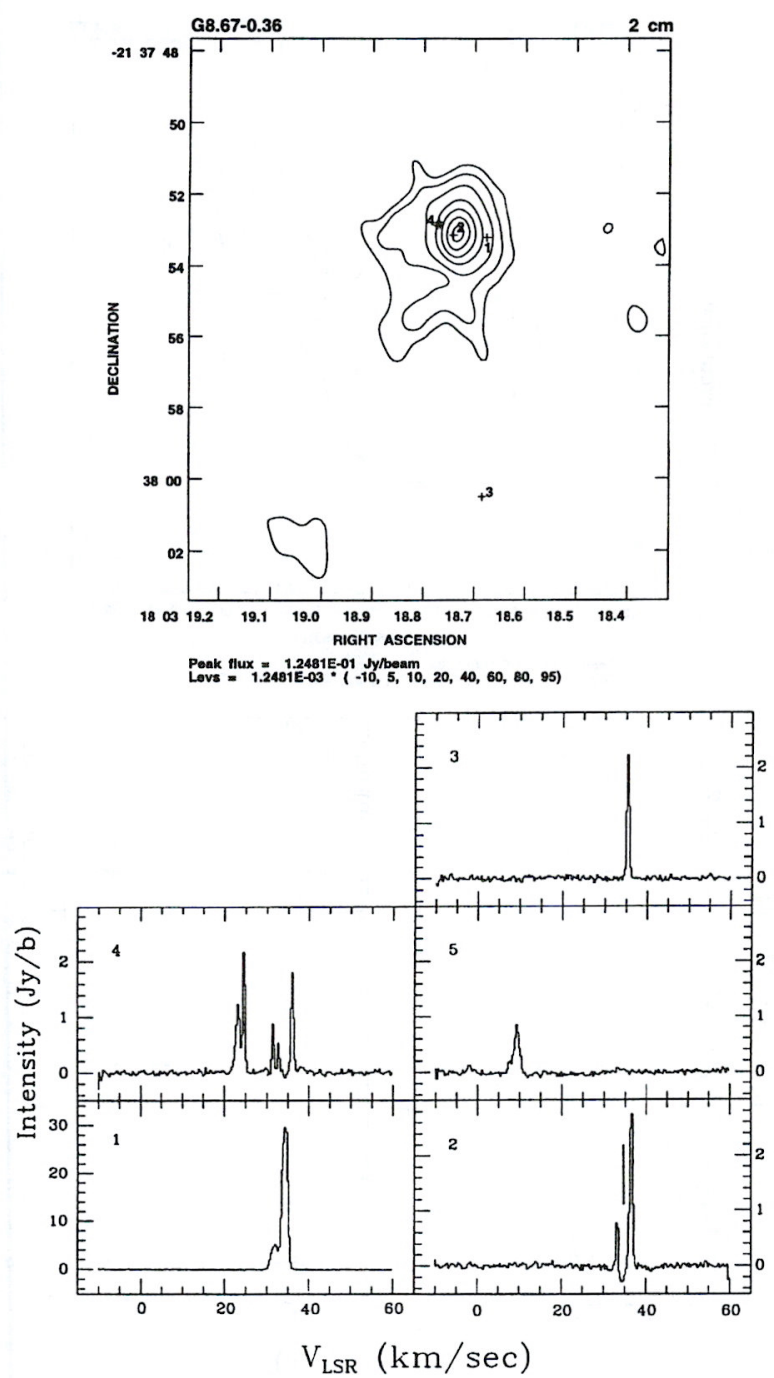

Fig. 11. Results for G8.67-0.36. Captions are as in Fig. 1

3.8. $G 34.26+0.15$

G34.26+0.15 is a UC HII region with a cometary morphology. Very strong maser emission is detected in front of the cometary arc embedded in an ultradense molecular core with temperature $225 \mathrm{~K}$ and a hydrogen density $10^{7.2} \mathrm{~cm}^{-3}$ (Henkel et al. 1987; Heaton et al. 1989). Also embedded in this ultradense molecular core are two unresolved continuum sources which show a spectral behavior indicative of an ionization bounded stellar wind (Gaume et al. 1994); however none of the maser clusters detected here is coincident with these sources. The strongest maser emission (cluster 1 in Fig. 5) comes from an elongated structure, oriented perpendicular to the cometary arc of the UC HII region.

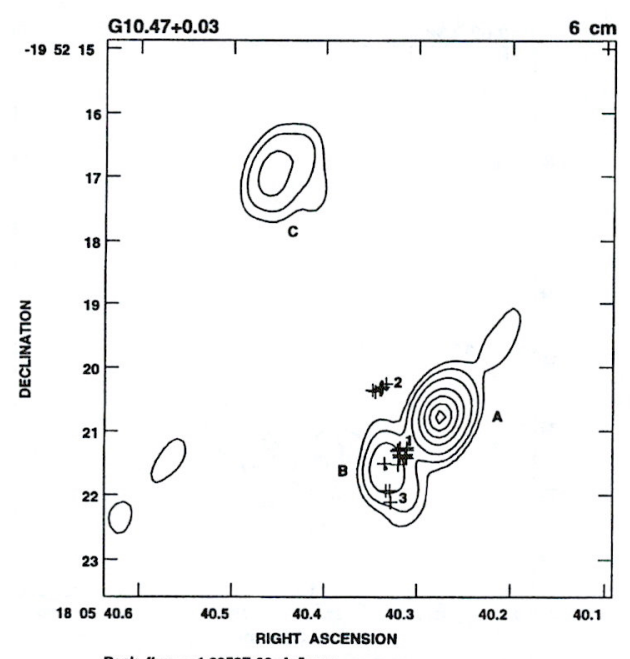

Peak flux $=1.6956 \mathrm{E}-02$
Levs $=1.6956 \mathrm{E}-04$
$-10,5,10,20,40,60,80,95)$

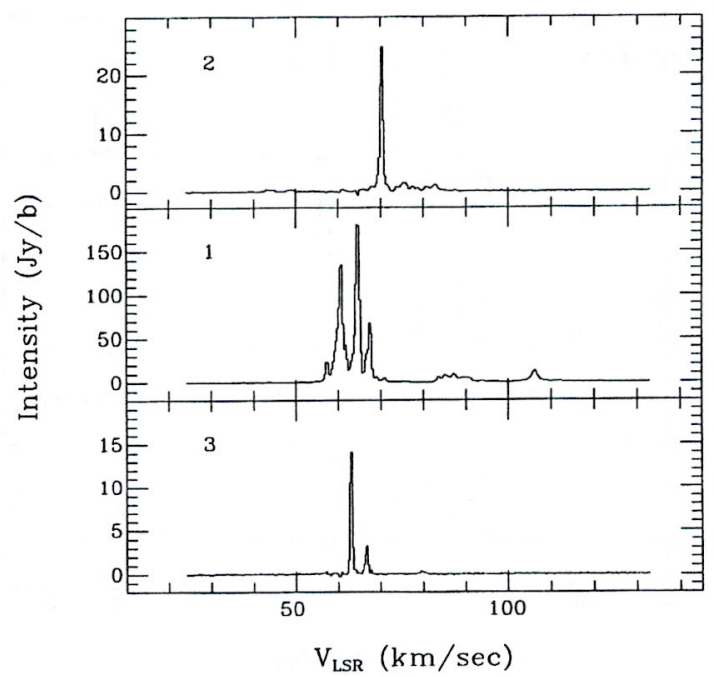

Fig. 12. Results for G10.47+0.03. Captions are as in Fig. 1. We have indicated continuum components $\mathrm{A}, \mathrm{B}$ and $\mathrm{C}$, using WC's nomenclature

\section{9. $G 45.07+0.13$}

Recent interferometric observations of $\operatorname{CS}(J=2-1)$ by Hunter et al. (1995) toward this region revealed a bipolar molecular flow centered on the UC HII region, and oriented with a P.A. of about $-45^{\circ}$. A comparison with our $\mathrm{H}_{2} \mathrm{O}$ maser data shows that maser clusters 1,3 and 4 in G45.07+0.13 are located along the flow axis and also show a good correspondence in the measured velocities. Hence, as in the case of G5.89-0.39, there is strong evidence that in $\mathrm{G} 45.07-0.13$ the $\mathrm{H}_{2} \mathrm{O}$ masers are taking part in the bipolar outflow. 

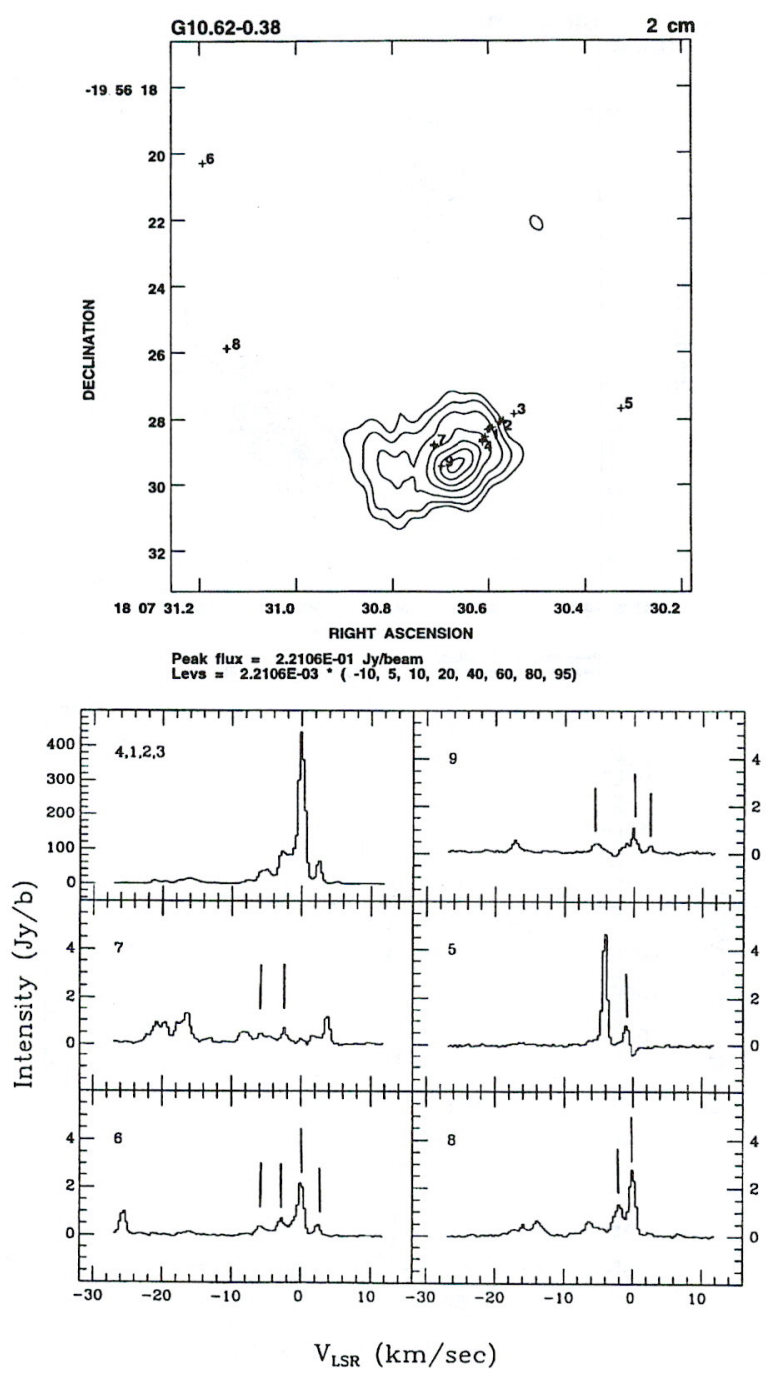

Fig. 13. Results for G10.62-0.38. Captions are as in Fig. 1

\section{The relationship between $\mathrm{H}_{2} \mathrm{O}$ masers and $\mathrm{UC}$ HII regions}

In order not to confuse the relationship between $\mathrm{H}_{2} \mathrm{O}$ maser emission and UC HII regions in the analysis presented in this section, we have excluded sources which have more than one compact HII region as reported in WC and Kurtz et al. 1994 (hereafter KCW). Note, however that these surveys were not sensitive to larger HII regions, which could, and in some cases (e.g. G34.26+0.15) are known to be present. We are left with 15 sources which have a total of 76 centers of maser emission.

Figures 22a-c shows histograms of the projected angular distance of the centers of water maser emission from the radio continuum peak of the UC HII regions and Figs. 23a-c shows the corresponding linear projected distance. About $8 \%$ of the maser clusters appear outside of the ranges displayed in these figures i.e. at angular sepa-
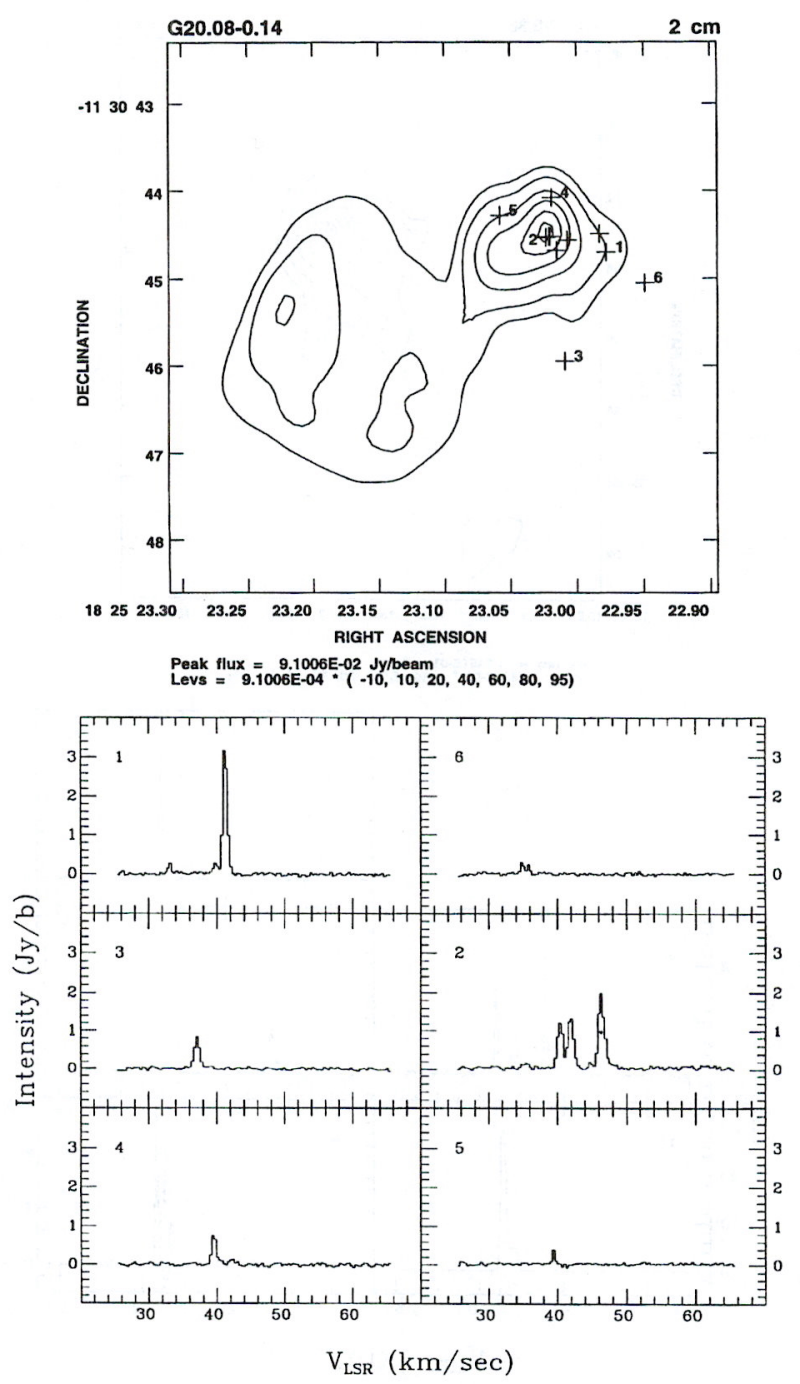

Fig. 14. Results for G20.08-0.14. Captions are as in Fig. 1

ration $>20^{\prime \prime}$ or projected linear distance $>0.5 \mathrm{pc}$ and are likely to be associated with different centers of star formation. The median values of these distributions are 2 .'9 and $0.096 \mathrm{pc}$ for the angular distance and projected linear distance, respectively. With a typical radius of $2^{\prime \prime}$ for the UC HII regions, it is clear from these histograms that most maser features are not observed projected onto the ionized gas. However, the small median distance of about $0.1 \mathrm{pc}$ indicates that most $\mathrm{H}_{2} \mathrm{O}$ masers are likely associated with the same molecular core as the UC HII region.

Inspecting Figs. 10-19 one notes that often maser features appear projected onto the radio continuum contours in the case of non-cometary morphologies. Figures 22 and 23 (middle and lower panels) show the distribution of angular and linear distances between maser features and ionized gas for cometary and non-cometary morphologies. The distribution in the case of non-cometary morphologies (Fig. 23c) is strongly peaked at a distance of 


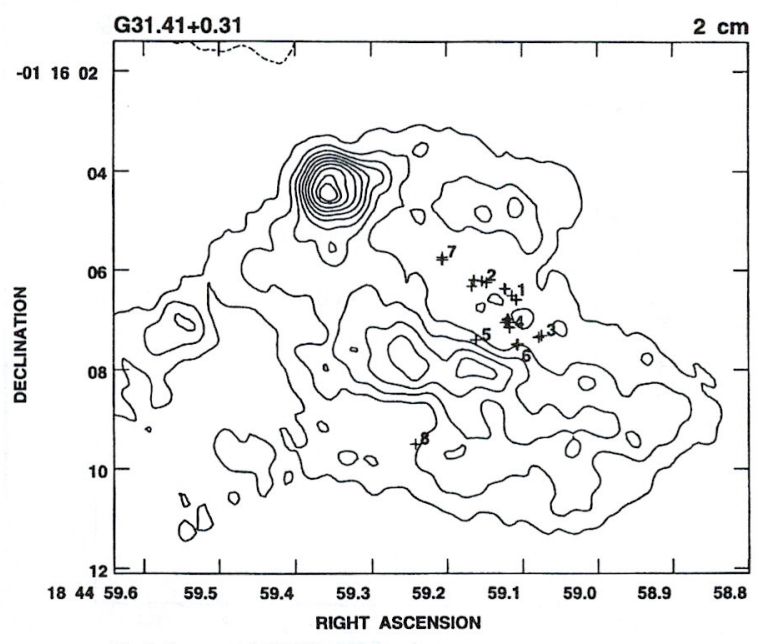

Peak flux $=9.4365 \mathrm{E}-03$ Jy/beam
Levs $=9.4365 \mathrm{E}-05 \cdot(-20,-10,10,20,30,40,50,60,70,80,90,95)$

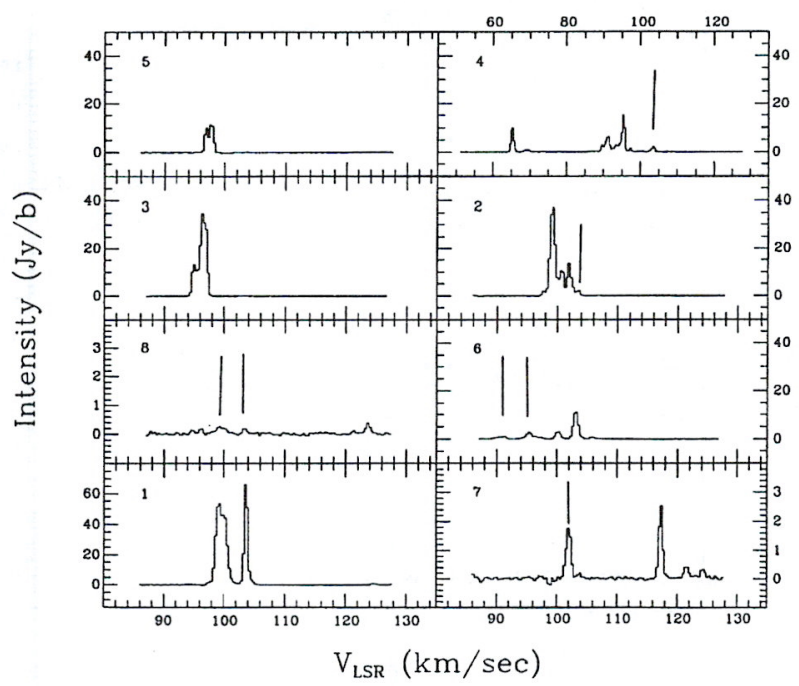

Fig. 15. Results for G31.41+0.31. Captions are as in Fig. 1. Note the different velocity scale for the spectrum of maser feature No. 4 in Fig. 15 b

$0.03 \mathrm{pc}$ whereas the distribution in the case of cometary features is flat (Fig. 23b). Also from inspection of Figs. 19 , one notes that in the case of the cometary morphologies, water maser emission is found predominantly to be located in front of the cometary arc. This can be seen clearly in the following sources: G11.94-0.62, G12.21-0.10, G19.61-0.23, G29.96-0.02, G34.26+0.15 and G75.78-0.34. Sources, where this is less clear are G43.89-0.38, where the maser emission is located to the side of UC HII region, G32.80+0.19, where our continuum map has too low signal-to-noise ratio (but compare KCW's map) and G9.62+0.19, where the cometary is the extended continuum component B (see Garay et al. 1993), which is not imaged well in our map. In G35.20-1.74 the water maser emission is not associated with the cometary UC HII region. Thus, including also the confused regions, we

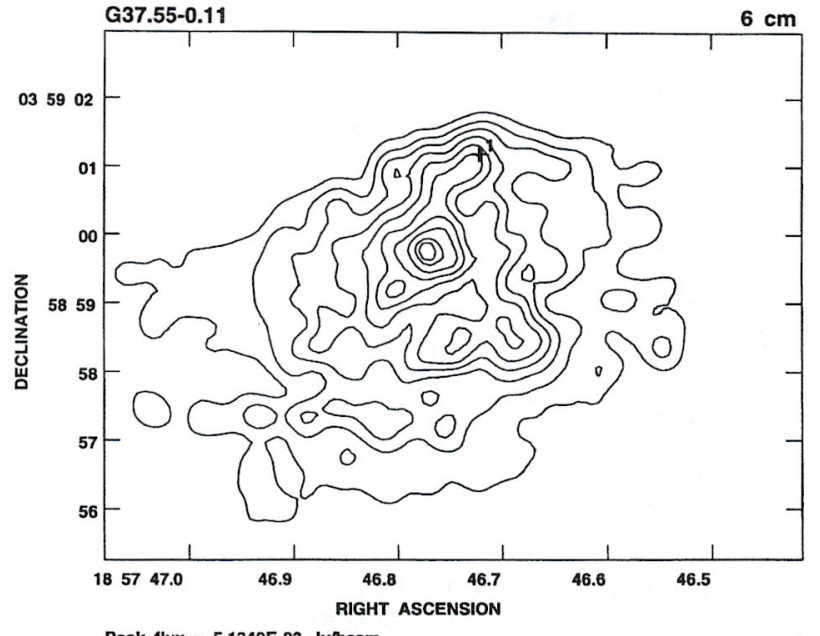

Peak flux $=5.1340 \mathrm{E}-03$ Jy/beam
Levs $=5.1340 \mathrm{E}-05 *(-10,10,20,30,40,50,60,70,80,90,95)$

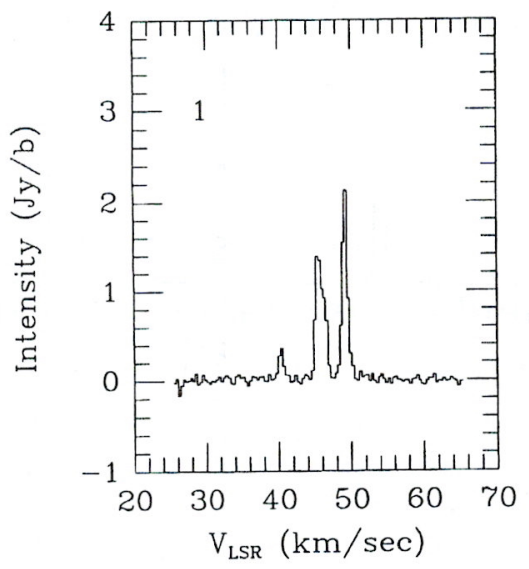

Fig. 16. Results for G37.55-0.11. Captions are as in Fig. 1

find water maser emission located in front of the cometary arc for 9 out of the 10 cometary regions observed. Also, several additional examples can be found in the literature, e.g. the compact HII region G351.42+0.64 (NGC 6334F) from Forster 1992.

One possible explanation for this dichotomy can be found in the bow shock theory of UC HII regions (e.g. van Buren et al. 1990). In this scenario a bow shock results from the relative movement of a young massive star and the surrounding dense molecular gas. The ionized gas exists in a shell which is held in a stable equilibrium between the inside pressure caused by the stellar wind and the outside ram pressure due to movement through the dense environment. Numerical modeling results by Mac Low et al. 1991 suggest that UC HII regions with a non-cometary morphology (spherical or core-halo) could be cometary regions observed head-on or tail-on, nearly parallel to the line of motion, so that water masers which are preferentially located in front of the ionized gas will be observed projected onto the ionized gas. 


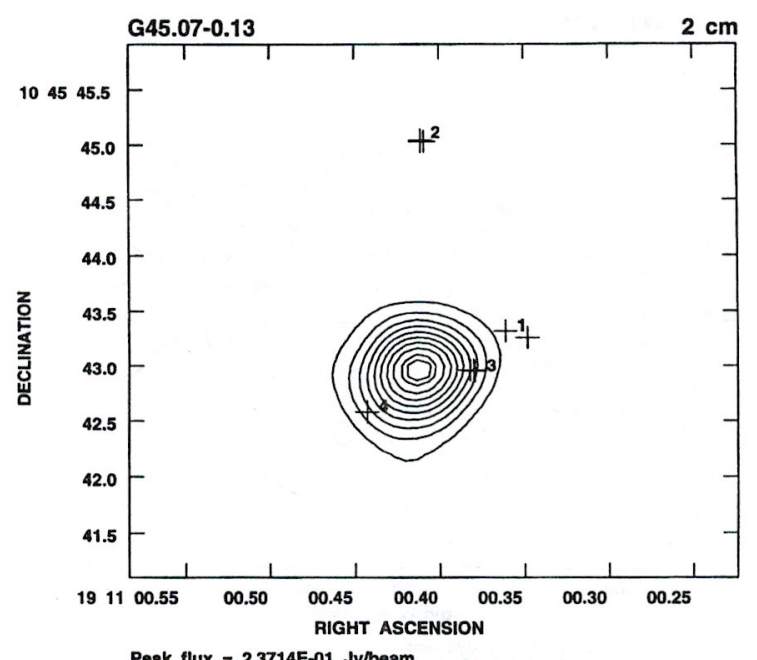

Peak flux $=2.3714 \mathrm{E}-01$ Jy/beam
Levs $=2.3714 \mathrm{E}-03 \cdot(-10,10,20,30,40,50,60,70,80,90,95)$

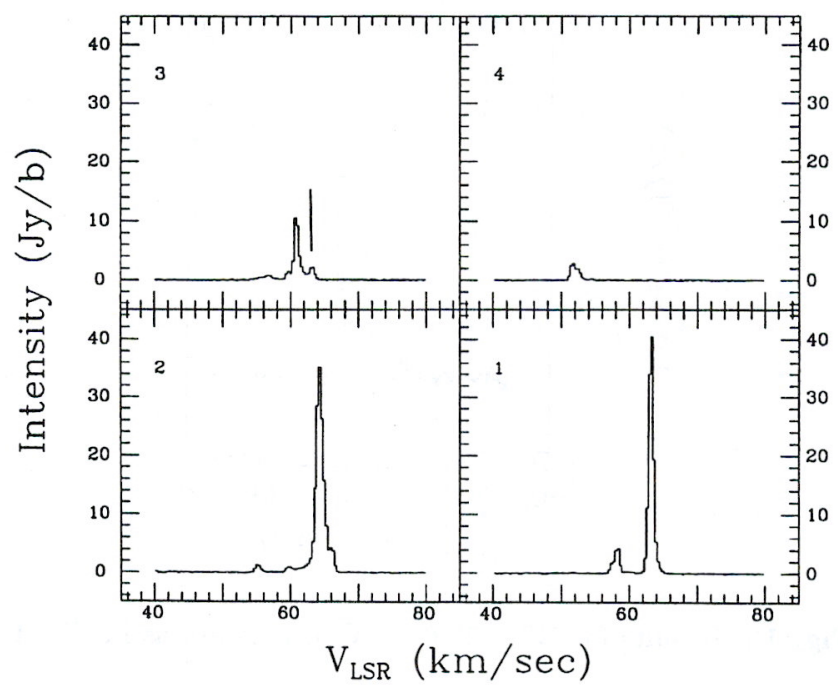

Fig. 17. Results for G45.07-0.13. Captions are as in Fig. 1

Within the frame work of the Bow-Shock theory, one might then ask whether the water masers are actually forming in the warm, compressed gas in the molecular bow shock, as was suggested for the $\mathrm{OH}$ masers surrounding the cometary UC HII region G34.26+0.15 (Churchwell 1991). The thickness of this layer of warm, shocked gas is predicted to be $\delta=\frac{9}{8} M^{-2} l$ where $M$ and $l$ are the Mach number and stand-off distance of the massive star from the shockfront (Van Buren et al. 1990). Adopting for $l 4 / 3$ of the distance between vertex and focus of the cometary parabola as listed in WC and assuming a Mach number of $M=5$ we find that the thickness of the shocked layer is predicted to be smaller than $0.01 \mathrm{pc}$ for all our sources. Comparing this with Fig. 23b we conclude that the water masers are located too far from the cometary arc of the ionized gas to be produced in the bow-shock. Also, in no case have we observed a distribution of water masers which follows closely the cometary arc as should be expected if
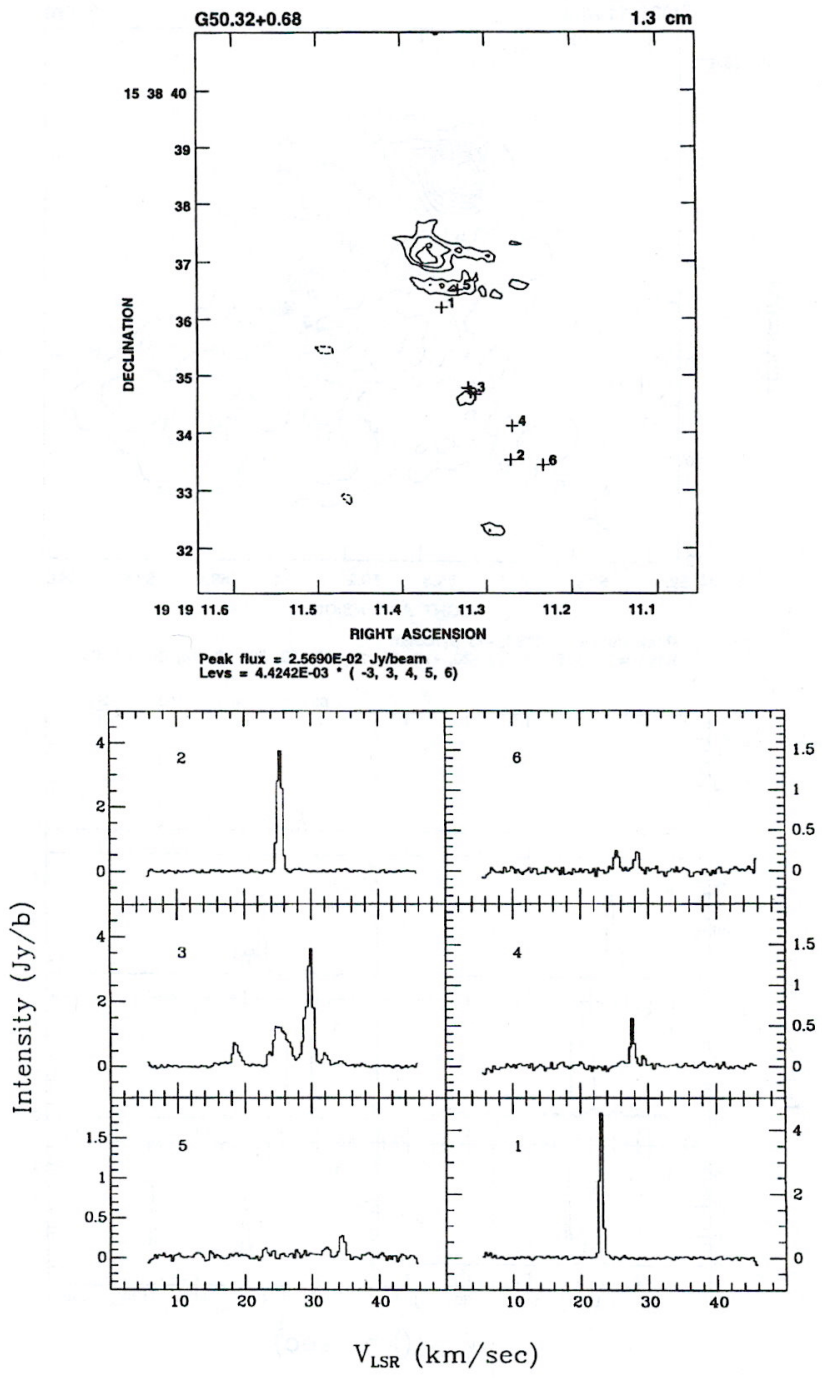

Fig. 18. Results for G50.32-0.10. Captions are as in Fig. 1. The continuum image was derived from line free channels in the $\mathrm{H}_{2} \mathrm{O}$ maser observations

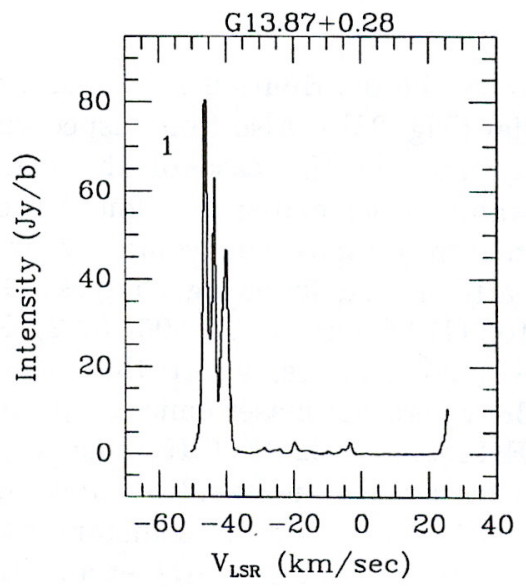

Fig. 19. Spectrum for maser cluster 1 in G13.87+0.28. For a continuum image, see Garay et al. 1993 


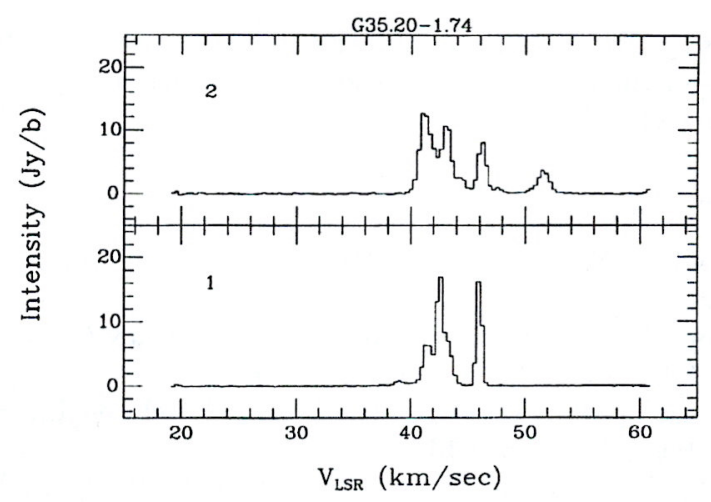

Fig. 20. Spectra for maser clusters 1 and 2 in G35.20-1.74. The centers of maser emission are at distances $>15^{\prime \prime}$ from the UC HII region

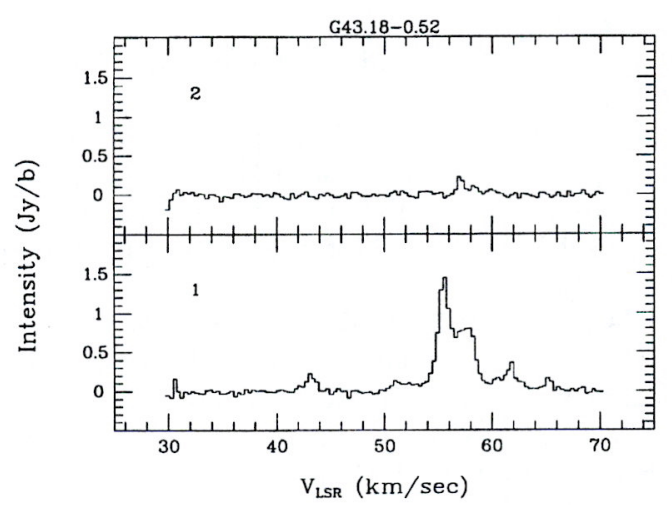

Fig. 21. Spectra for maser clusters 1 and 2 in G43.18-0.52. For a continuum image, see WC

the water masers are formed in the Bow-Shock. Hence, we conclude that it is unlikely that the $\mathrm{H}_{2} \mathrm{O}$ masers in front of the cometary regions observed in this survey are produced in the Bow-Shock. Gaume et al. 1994 have reached the same conclusion for the water masers observed toward G34.26+0.15.

Alternatively, the locations of the water masers could mark the positions of young, possible protostellar objects. Recently, CCHWK mapped emission in the $\mathrm{NH}_{3}(4,4)$ line toward G9.62+0.19, G10.47+0.03, G29.96-0.02 and G31.41+0.31. This study revealed hot $(T>100 \mathrm{~K})$, dense $\left(n\left(\mathrm{H}_{2}\right) \approx 10^{7} \mathrm{~cm}^{-3}\right)$ molecular clumps in the immediate vicinity of the UC HII regions. The most likely explanation for these objects are young massive objects, which are heating the surrounding molecular envelope. A comparison of $\mathrm{NH}_{3}(4,4)$ emission with our $\mathrm{H}_{2} \mathrm{O}$ maser data shows, that in the four cases studied, the $\mathrm{H}_{2} \mathrm{O}$ masers are coincident with the hot molecular gas. A similar coincidence of the $\mathrm{H}_{2} \mathrm{O}$ masers with hot, dense molecular gas is known for G10.62-0.38 (Keto et al. 1987, 1988), G34.26+0.15 (Heaton et al. 1989) and $\mathrm{W} 3\left(\mathrm{H}_{2} \mathrm{O}\right)$ (Turner \& Welch 1984). Thus, if most of the $\mathrm{H}_{2} \mathrm{O}$ masers observed

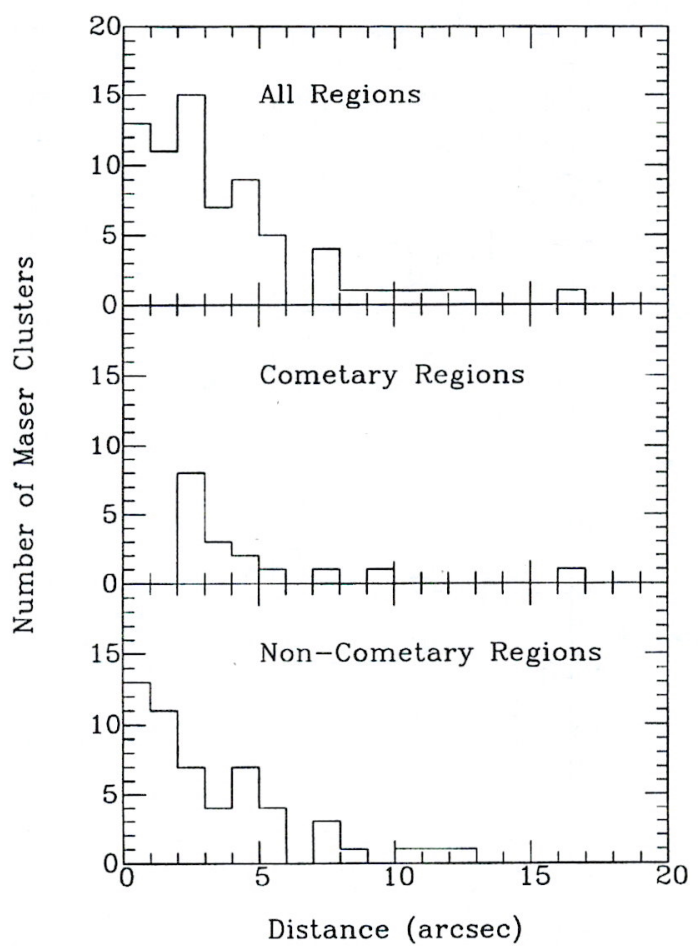

Fig. 22. Distribution of observed angular distances between $\mathrm{H}_{2} \mathrm{O}$ maser clusters and radio continuum peak. a) Top panel: All regions. b) Middle panel: Regions with cometary morphology. c) Lower panel: Regions with non-cometary morphology

in front of cometary HII regions, mark the location of deeply embedded, young massive stars, the fact that we observe centers of $\mathrm{H}_{2} \mathrm{O}$ masers toward positions in front of the cometary head implies, that massive star formation preferably occurs in a binary (or multiple) mode. The different appearance (UC HII region versus hot molecular clump) may be explained by either different ages, spectral types or physical environment. In the latter case, one could speculate that the cometary appearence of the ionized gas is caused by a gradient in a lower density environment, whereas higher densities prevent the rapid expansion and formation of an UC HII region at the position of the hot molecular clumps coincident with the water masers (see also De Pree et al. 1995).

\section{Summary}

In this paper we present high resolution images and spectra toward $21 \mathrm{H}_{2} \mathrm{O}$ maser sources in the vicinity of UC HII regions. This survey provides the basis for future VLBI studies of the maser clusters mapped in this paper. Emission from the $6_{16}-5_{23}$ masing transition of interstellar $\mathrm{H}_{2} \mathrm{O}$ is observed in the close vicinity of UC HII regions with a median angular distance of $2^{\prime \prime} 9$ and a median linear projected distance of $0.1 \mathrm{pc}$ from the continuum peak. We find that for UC HII regions with cometary 


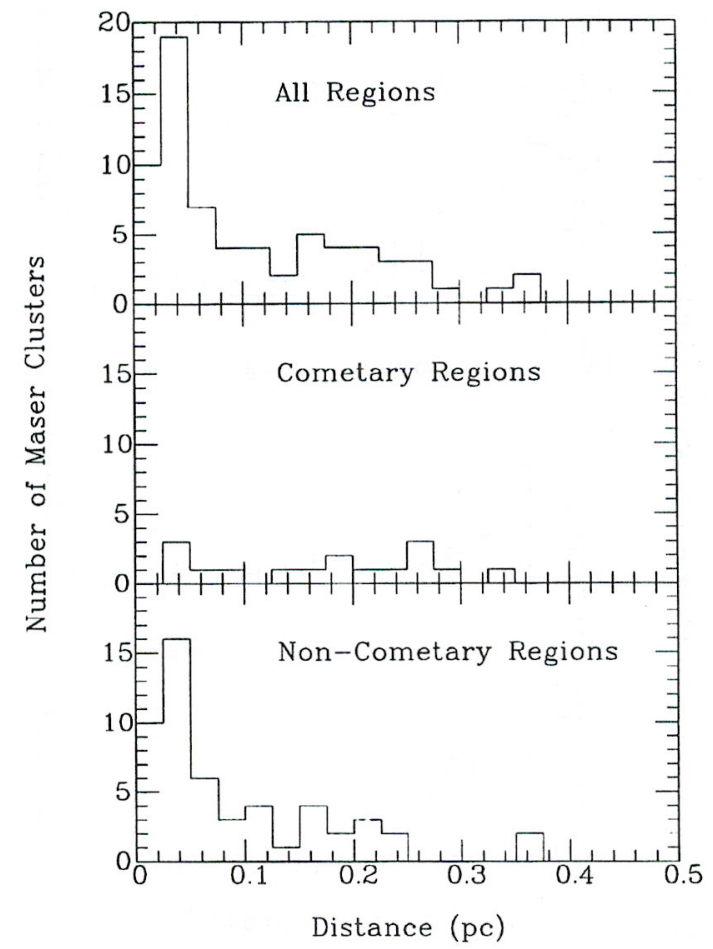

Fig. 23. Distribution of projected linear distances between $\mathrm{H}_{2} \mathrm{O}$ maser clusters and radio continuum peak. a) Top panel: All regions. b) Middle panel: Regions with cometary morphology. c) Lower panel: Regions with non-cometary morphology

morphology the water maser emission is located in front of the cometary arc whereas for non-cometary UC HII regions the water masers are often projected onto the ionized gas. Due to the large distance from the I-front of $>0.03 \mathrm{pc}$, it is unlikely, that the water masers are formed in the shocked layer of warm molecular gas in the interface between the ionized gas of the UC HII region and surrounding molecular gas as predicted by the Bow Shock theory of UC HII regions. A comparison with maps in the $\mathrm{NH}_{3}$ inversion transitions shows that in at least 7 cases the water masers are associated with hot $(T>100 \mathrm{~K})$, dense $\left(n\left(\mathrm{H}_{2}\right) \approx 10^{7} \mathrm{~cm}^{-3}\right)$ molecular clumps. For the UC HII regions G5.89-0.38 and G45.07+0.13 we find good spatial and velocity correspondence between water masers and outflowing molecular gas. It is thus likely that the $\mathrm{H}_{2} \mathrm{O}$ masers are taking part in the bipolar outflow.

Acknowledgements. We would like to thank Doug Wood for providing the continuum images in digital form and Malcolm
Walmsley for helpful discussions. Thanks are also due to the anonymous referee, whose suggestions improved the manuscript.

\section{References}

Cesaroni R., Walmsley C.M., Kömpe C., Churchwell E., 1991, A\&A 252, 278

Cesaroni R., Churchwell E., Hofner P., Walmsley C.M., Kurtz S., 1994, A\&A 288, 903 (CCHWK)

Cesaroni R., Olmi L., Walmsley C.M., Churchwell E., Hofner P., 1994, ApJ 435, L137

Churchwell E., Walmsley C.M., Cesaroni R., 1990, A\&AS 83, 119 (CWC)

Churchwell E., 1991, in The Physics of Early Stellar Evolution, In: Lada C.J. \& Kylafis N.D. (eds.) NATO ASI Ser. 342

Churchwell E., 1993 in Massive Stars: Their Lives in the Interstellar Medium. In: Cassinelli J.P. \& Churchwell E.B. (eds.) ASP Conf. Ser. 35

De Pree C.G., Rodriguez L.F., Goss W.M., 1995, Rev. Mex. A\&A 31,39

Elitzur M., Hollenbach D.J., McKee C.F., 1989, ApJ 346, 983

Forster J.R., 1992, in Astrophysical Masers. In: Clegg A.W. \& Nedoluha G.E. (eds.). Heidelberg: Springer, p. 108

Garay G., Rodriguez L.F., Moran J.M., Churchwell E., 1993, ApJ 418, 368

Gaume R.A., Mutel R.L., 1987, ApJS 65, 193

Gaume R.A., Fey A.L., Claussen M.J., 1994, ApJ 432, 648

Genzel R., Downes D., 1977, A\&AS 30, 145

Harvey P.M., Forveille T., 1988, A\&A 197, L19

Heaton B.D., Little L.T., Bishop I.S., 1989, A\&A 213, 148

Henkel C., Wilson T.L., Mauersberger R., 1987, A\&A 182, 137

Hofner P., Kurtz S., Churchwell E., Walmsley C.M., Cesaroni R., 1994, ApJ 429, L85

Hofner P., Kurtz S., Churchwell E., Walmsley C.M., Cesaroni R., 1996, ApJ 460, 359

Hunter T.R., Phillips T.G., Menten K., 1995, (in preparation)

Kaufman M.J., Neufeld D.A., 1995, ApJ 456, 250

Keto E., Ho P.T.P., Haschick A.D., 1987, ApJ 318, 712

Keto E., Ho P.T.P., Haschick A.D., 1988, ApJ 324, 920

Kurtz S., Churchwell E., Wood D.O.S., 1994, ApJS 91, 659 (KCW)

Mac Low M.-M., Wood D.O.S., Churchwell E., 1991, ApJ 369, 395

Tofani G., Felli M., Taylor G.B., Hunter T.R., 1995, A\&AS 112,299

Turner J.L., Welch W.J., 1984, ApJ 287, L81

Wood D.O.S., Churchwell E., 1989a, ApJ 340, 265

Wood D.O.S., Churchwell E., 1989b, ApJS 69, 831 (WC)

Van Buren D., Mac Low M.-M., Wood D.O.S., Churchwell E., 1990, ApJ 353, 570

Zijlstra A.A., Pottasch S.R., Engels D., Roelfsema P.R., te Lintel Hekkert P., Umana G., 1990, A\&A 246, 217 
Table 3. Observed water maser features

\begin{tabular}{|c|c|c|c|c|c|c|c|c|c|}
\hline \multirow[b]{2}{*}{ Source } & \multirow[b]{2}{*}{ no. } & \multicolumn{2}{|c|}{$\alpha(1950)$} & \multicolumn{3}{|c|}{$\delta(1950)$} & \multirow{2}{*}{$\begin{array}{c}\mathrm{V}_{\mathrm{LSR}} \\
\left(\mathrm{km} \mathrm{s}^{-1}\right)\end{array}$} & \multirow{2}{*}{$\begin{array}{c}\mathrm{I}_{\nu} \\
(\mathrm{Jy} / \mathrm{b})\end{array}$} & \multirow{2}{*}{$\begin{array}{c}\delta \mathrm{V} \\
\left(\mathrm{km} \mathrm{s}^{-1}\right)\end{array}$} \\
\hline & & (h min & in $\mathrm{sec}$ ) & $0^{0}$ & 1 & ") & & & \\
\hline \multirow[t]{9}{*}{ G5.89-0.38 } & 1 & 1757 & 26.709 & -24 & 03 & 48.30 & 78.4 & 62.1 & $5.9^{a}$ \\
\hline & 2 & & 26.818 & & 03 & 56.10 & 20.2 & 45.3 & 13.9 \\
\hline & 3 & & 26.701 & & 04 & 02.57 & -3.2 & 32.5 & 15.2 \\
\hline & 4 & & 26.674 & & 03 & 59.84 & 8.7 & 13.7 & $1.7^{a}$ \\
\hline & 5 & & 26.476 & & 04 & 00.68 & 8.7 & 11.6 & $2.3^{a}$ \\
\hline & 6 & & 26.744 & & 04 & 00.00 & 10.3 & 2.7 & $1.3^{a}$ \\
\hline & 7 & & 26.960 & & 03 & 55.68 & 18.2 & 0.6 & $1.0^{a}$ \\
\hline & 8 & & 26.996 & & 03 & 56.06 & 10.7 & 0.6 & $1.0^{a}$ \\
\hline & 9 & & 26.835 & & 03 & 59.24 & 9.7 & 0.3 & $1.0^{a}$ \\
\hline \multirow[t]{5}{*}{ G8.67-0.36 } & 1 & 1803 & 18.677 & -21 & 37 & 53.22 & 34.3 & 29.5 & 2.3 \\
\hline & 2 & & 18.743 & & 37 & 53.15 & 36.6 & 2.7 & 3.6 \\
\hline & 3 & & 18.684 & & 38 & 00.51 & 35.3 & 2.2 & $1.7^{a}$ \\
\hline & 4 & & 18.772 & & 37 & 52.80 & 24.5 & 2.1 & 12.8 \\
\hline & 5 & & 18.592 & & 37 & 40.94 & 9.3 & 0.9 & 11.1 \\
\hline \multirow[t]{9}{*}{ G9.62+0.19 } & 1 & 1803 & 16.064 & -20 & 32 & 02.00 & 5.0 & 75.0 & 2.0 \\
\hline & 2 & & 16.012 & & 31 & 58.22 & 5.3 & 16.6 & 19.1 \\
\hline & 3 & & 16.130 & & 32 & 02.35 & 6.0 & 9.5 & $3.0^{a}$ \\
\hline & 4 & & 16.057 & & 32 & 01.38 & 7.0 & 6.4 & 2.0 \\
\hline & 5 & & 15.979 & & 31 & 55.50 & -9.8 & 2.9 & 12.2 \\
\hline & 6 & & 16.071 & & 31 & 58.36 & 6.0 & 2.2 & 19.7 \\
\hline & 7 & & 16.082 & & 31 & 59.18 & 7.3 & 1.5 & $2.0^{a}$ \\
\hline & 8 & & 15.867 & & 31 & 52.22 & 0.1 & 1.3 & 2.0 \\
\hline & 9 & & 16.175 & & 32 & 03.33 & 25.1 & 0.3 & $1.7^{a}$ \\
\hline \multirow[t]{3}{*}{$\mathrm{G} 10.47+0.03$} & 1 & 1805 & 40.321 & -19 & 52 & 21.30 & 64.4 & 202.1 & 83.9 \\
\hline & 2 & & 40.340 & & 522 & 20.34 & 70.3 & 27.9 & 39.8 \\
\hline & 3 & & 40.329 & & 522 & 21.93 & 63.1 & 14.4 & 16.4 \\
\hline \multirow[t]{9}{*}{ G10.62-0.38 } & 1 & 1807 & 30.600 & -19 & 56 & 28.30 & -0.1 & 237.1 & 6.3 \\
\hline & 2 & & 30.569 & & 562 & 28.02 & -0.1 & 205.5 & 7.4 \\
\hline & 3 & & 30.547 & & 56 & 27.82 & -1.7 & 36.7 & $5.9^{a}$ \\
\hline & 4 & & 30.607 & & 56 & 28.52 & -2.7 & 36.0 & 30.0 \\
\hline & 5 & & 30.326 & & 56 & 27.66 & -4.1 & 6.0 & $2.0^{a}$ \\
\hline & 6 & & 31.193 & & 56 & 20.30 & -25.4 & 1.4 & $2.0^{a}$ \\
\hline & 7 & & 30.713 & & 56 & 28.77 & -16.2 & 1.3 & 24.6 \\
\hline & 8 & & 31.143 & & 56 & 25.88 & -6.4 & 1.0 & 9.5 \\
\hline & 9 & & 30.698 & & 56 & 29.44 & -17.2 & 0.6 & $2.0^{a}$ \\
\hline \multirow[t]{3}{*}{ G11.94-0.62 } & $1^{b}$ & 1811 & 01.473 & -18 & 55 & 14.87 & 40.7 & 248.1 & 16.4 \\
\hline & 2 & & 04.256 & & 54 & 20.99 & 44.7 & 5.2 & $1.7^{a}$ \\
\hline & 3 & & 03.701 & & 54 & 18.41 & 16.0 & 3.9 & 1.7 \\
\hline \multirow[t]{8}{*}{$\mathrm{G} 12.21-0.10$} & 1 & 1809 & 43.897 & -18 & 25 & 06.68 & 20.1 & 116.9 & 17.4 \\
\hline & 2 & & 43.763 & & 25 & 06.70 & -4.5 & 111.0 & 78.3 \\
\hline & $3^{b}$ & & 48.455 & & 25 & 14.23 & 26.4 & 70.4 & 9.2 \\
\hline & 4 & & 43.823 & & 25 & 07.74 & 23.4 & 3.2 & $1.7^{a}$ \\
\hline & 5 & & 43.794 & & 25 & 05.80 & 31.7 & 1.5 & 13.4 \\
\hline & $6^{b}$ & & 48.458 & & 25 & 13.72 & 28.4 & 2.3 & 4.6 \\
\hline & 7 & & 43.864 & & 25 & 05.51 & 8.0 & 0.5 & $1.3^{a}$ \\
\hline & 8 & & 44.136 & & 25 & 05.49 & -17.7 & 0.4 & 10.5 \\
\hline
\end{tabular}


Table 3. continued

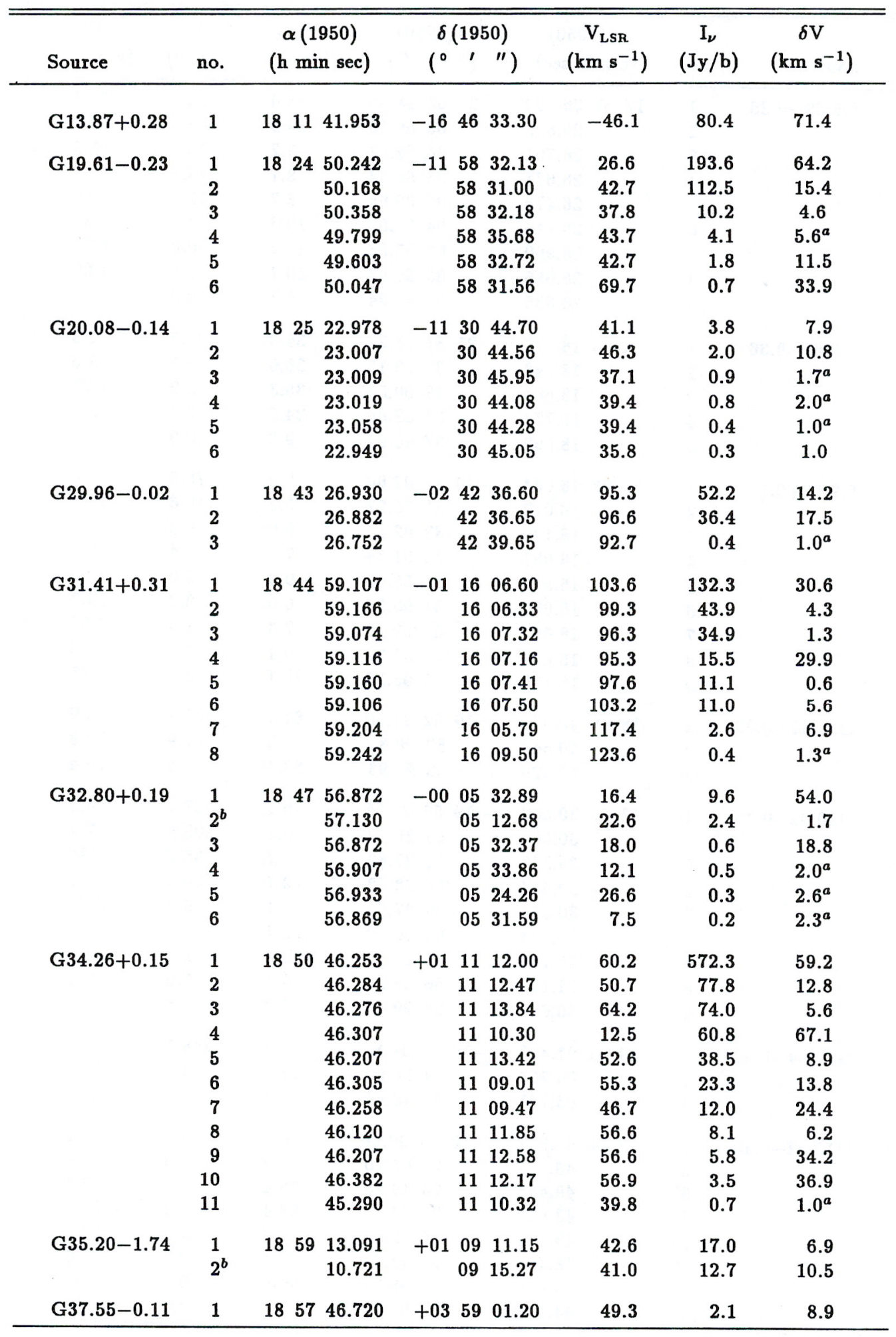


Table 3. continued

\begin{tabular}{|c|c|c|c|c|c|c|c|c|c|}
\hline \multirow[b]{2}{*}{ Source } & \multirow[b]{2}{*}{ no. } & \multicolumn{2}{|c|}{$\alpha(1950)$} & \multicolumn{3}{|c|}{$\delta(1950)$} & \multirow{2}{*}{$\begin{array}{c}\mathrm{V}_{\mathrm{LSR}} \\
\left(\mathrm{km} \mathrm{s}^{-1}\right)\end{array}$} & \multirow{2}{*}{$\begin{array}{c}\mathrm{I}_{\nu} \\
(\mathrm{Jy} / \mathrm{b})\end{array}$} & \multirow{2}{*}{$\begin{array}{c}\delta \mathrm{V} \\
\left(\mathrm{km} \mathrm{s}^{-1}\right)\end{array}$} \\
\hline & & (h mi & in sec) & $0^{0}$ & 1 & ") & & & \\
\hline \multirow[t]{2}{*}{ G43.18-0.52 } & 1 & 1909 & 45.323 & +08 & 47 & 09.81 & 55.6 & 1.6 & 22.0 \\
\hline & 2 & & 45.130 & & 47 & 08.72 & 56.9 & 0.2 & $0.7^{a}$ \\
\hline \multirow[t]{2}{*}{ G43.89-0.78 } & 1 & 1912 & 02.990 & +09 & 17 & 21.30 & 46.8 & 2.8 & $3.0^{a}$ \\
\hline & 2 & & 03.032 & & 17 & 21.81 & 53.0 & 0.6 & 5.6 \\
\hline \multirow{4}{*}{$\mathrm{G} 45.07+0.13$} & 1 & 1911 & 00.361 & +10 & 45 & 43.32 & 63.3 & 45.4 & 5.3 \\
\hline & 2 & & 00.409 & & 45 & 45.03 & 64.3 & 37.5 & 10.8 \\
\hline & 3 & & 00.379 & & 45 & 42.95 & 60.7 & 10.5 & 4.0 \\
\hline & 4 & & 00.443 & & 45 & 42.58 & 51.8 & 2.8 & $3.3^{a}$ \\
\hline \multirow{6}{*}{ G50.32+0.68 } & 1 & 1919 & 11.352 & +15 & 38 & 36.20 & 23.0 & 4.5 & $1.3^{a}$ \\
\hline & 2 & & 11.271 & & 38 & 33.53 & 25.3 & 3.8 & $1.7^{a}$ \\
\hline & 3 & & 11.317 & & 38 & 34.71 & 29.6 & 3.1 & 15.8 \\
\hline & 4 & & 11.269 & & 38 & 34.12 & 27.6 & 0.6 & $1.3^{a}$ \\
\hline & 5 & & 11.333 & & 38 & 36.51 & 34.5 & 0.3 & $1.0^{a}$ \\
\hline & 6 & & 11.233 & & 38 & 33.44 & 28.3 & 0.2 & $1.0^{a}$ \\
\hline \multirow[t]{5}{*}{ G75.78-0.34 } & 1 & 2019 & 51.880 & +37 & 17 & 00.31 & 2.6 & 58.4 & 34.5 \\
\hline & 2 & & 51.888 & & 16 & 59.99 & 2.3 & 40.2 & 37.9 \\
\hline & $3^{b}$ & & 48.968 & & 15 & 52.00 & -7.9 & 66.2 & 11.8 \\
\hline & 4 & & 51.918 & & 16 & 59.96 & 13.5 & 4.2 & 30.9 \\
\hline & $5^{b}$ & & 49.221 & & 16 & 00.77 & -3.9 & 4.3 & $1.7^{a}$ \\
\hline
\end{tabular}

${ }^{a}$ Full Width at Zero Power for single features

b Distance to UC HII region $>20^{\prime \prime}$ 